\title{
RNA-Seq Transcriptome Analysis of Pronounced Biconcave Vertebrae: A Common Abnormality in Rainbow Trout (Oncorhynchus mykiss, Walbaum) Fed a Low-Phosphorus Diet
}

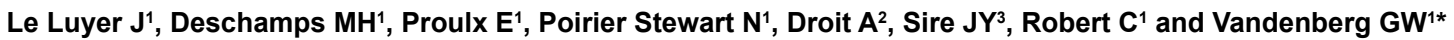 \\ ${ }^{1}$ Département of des sciences animales, Pavillon Paul-Comtois, Université Laval, Québec, QC, Canada G1V 0A6, Canada \\ ${ }^{2}$ Department of Molecular Medicine, Centre de Recherche du CHU de Québec, Université Laval, Québec, QC, G1V 4G2 Canada \\ ${ }^{3}$ Institut de Biologie Paris-Seine, UMR 7138-Evolution Paris-Seine, Université Pierre et Marie Curie, Paris, France
}

\begin{abstract}
The prevalence of bone deformities, particularly linked with mineral deficiency, is an important issue for fish production. Juvenile triploid rainbow trout (Oncorhynchus mykiss) were fed a low-phosphorus (P) diet for 27 weeks (60 to $630 \mathrm{~g}$ body mass). At study termination, $24.9 \%$ of the fish fed the low-P diet displayed homogeneous biconcave vertebrae (deformed vertebrae phenotype), while 5.5\% displayed normal vertebral phenotypes for the entire experiment. The aim of our study was to characterize the deformed phenotype and identify the putative genes involved in the appearance of $\mathrm{P}$ deficiency-induced deformities. Both $\mathrm{P}$ status and biomechanical measurements showed that deformed vertebrae were significantly less mineralized $(55.0 \pm 0.4$ and $59.4 \pm 0.5, \%$ ash DM, for deformed and normal vertebrae, respectively) resulting in a lower stiffness $(80.3 \pm 9.0$ and $140.2 \pm 6.3 \mathrm{~N} / \mathrm{mm}$, for deformed and normal phenotypes, respectively). The bone profiles based on $\mu \mathrm{CT}$ observations showed no difference in the osteoclastic resorption while no difference in matrix production was observed between deformed (total bone area $5442.0 \pm 110.1 \mu \mathrm{m}^{2}$ ) and normal vertebrae (total bone area $5931.2 \pm 249.8 \mu \mathrm{m}^{2}$ ) in this study. Consequently, the lower $\mathrm{P}$ content rather results from a reduced degree of mineralization in the deformed phenotype. Finally, we quantified differential gene expression between deformed vertebrae (pronounced biconcave) and normal phenotype by employing deep RNA-sequencing and mapping against a reference bone transcriptome for rainbow trout. In total, 1289 genes were differentially expressed. Among them, in deformed fish we observed that BGLAP, MGP and NOG, an inhibitor of BMP signalling pathway, were up-regulated while COL11a1 was down-regulated. These genes are central actors involved in the reduced degree of mineralization triggering vertebral deformities. These results will further the understanding of $P$ deficiency-induced deformities; hence providing new tools for improved $P$ management in production settings.
\end{abstract}

Keywords: RNA-sequencing; Deformities; Rainbow trout; Phosphorus

Abbreviations: BGLAP: Osteocalcin; BMPR2: Bone Morphogenetic Protein Receptor, type II; CEBPB: CCAAT/Enhancer Binding Protein (C/EBP) B; COL11a1: Collagen type XI alpha 1; COL12a1: Collagen type X2 alpha 1, CREBBP; CREB Binding Protein; DCHS1: Dachsous Cadherin-Related 1; FAT4: FAT Atypical Cadherin 4; FBN2: Fibrillin; FGFR4: Fibroblast Growth Factor Receptor 4; FLNA: Filamin A, alpha; GCG2: Glucagon-2; GPC1: Glypican 1; HIVEP3: Human Immunodeficient Virus Type 1 Enhancer Binding Protein 3; HSPG2: Heparan Sulfate Proteoglycan 2; JUNB: Jun B proto-oncogene; JUND: Jun D proto-oncogene; LRP6: Low density lipoprotein receptor-Related Protein 6; M3K1: Mitogen-Activated Protein Kinase Kinase Kinase 1; MEF2C: Myocyte-Specific Enhancer Factor 2C; MGP: Matrix Gla Protein; MMP10: Matrix Metallopeptidase 10; MYCB2: Myc Binding Protein 2; MYCPP: C-myc Promoter-Binding Protein; MYO10: Unconventional Myosin-X; NF1: Neurofibromin 1; NFIX: Nuclear Factor 1/X (CCAAT-binding transcription factor); NIK: MitogenActivated Protein Kinase Kinase Kinase 4; NKX3.2: Homeobox Protein Nkx3.2; NOG: Noggin; OPG: Osteoprotegerin; SFRP1: Secreted Frizzled-Related Protein 1; SHH: Sonic Hedgehog; SOS1: Son of Sevenless Homolog 1; SOX9: Sex Determining Region Y-Box 9; SPARC: Osteonectin; SPP1: Osteopontin; SRCAP: nf2-Related CREBBP Activator Protein; TNR5: CD40 molecule TNF Receptor Family Member 5; TRAP: Tartrate Resistant Alkaline Phosphatase; TMEFF1: Tomoregulin-1; TWIST2: Twist Family BHLH Transcription Factor 2; USP9X: Probable Ubiquitin Carboxyl-Terminal Hydrolase FAF-X; VDR: Vitamin D Receptor; VGFR1: Vascular Endothelial Growth Factor Receptor 1

\section{Introduction}

Extensive work regarding deformity development in teleost fish have revealed that biotic and abiotic factors affect this condition including genetics, ploidy as well as nutrition [1-3]. Among the main nutrients affecting skeletal development in fish, phosphorus (P) particularly has drawn particular attention [4-6]. Bone tissue mineral (hydroxyapatite) constitutes the main reservoir of $\mathrm{P}$, comprising up to $85 \%$ of the total body content $[7,8]$. To date, many studies in salmonids have attempted to identify and characterize the response of the fish skeleton to low-P diets. Under extreme conditions such as reproduction, migration or starvation, teleosts mobilize P from scales and bone tissues [9-13]. During a prolonged $\mathrm{P}$ deficiency, fish may also display lower growth performance [14], lower mineral status of bone, scales and flesh $[3,15,16]$ and, in some severe cases, increased occurrence of specific phenotypes of vertebral deformities [17-20].

The study of vertebral abnormalities is particularly difficult in regard to the complexity and the role of the axial skeleton. In trout, this system is comprised of 64 vertebrae, composed of several tissues

*Corresponding author: Grant Vandenberg, Département des sciences animales, Université Laval, Pavillon Paul-Comtois, local 4211, 2425, rue de l'Agriculture, Québec, QC, G1V 0A6, Canada, Tel: (001)(418) 656-2131 \# 6541; Fax: (001)(418) 656-3766; E-mail: Grant.Vandenberg@fsaa.ulaval.ca

Received February 05, 2015; Accepted April 22, 2015; Published April 24, 2015

Citation: Le Luyer J, Deschamps MH, Proulx E, Poirier Stewart N, Droit A, et al. (2015) RNA-Seq Transcriptome Analysis of Pronounced Biconcave Vertebrae: A Common Abnormality in Rainbow Trout (Oncorhynchus mykiss, Walbaum) Fed a Low-Phosphorus Diet. Next Generat Sequenc \& Applic 2: 112. doi:10.4172/2469-9853.1000112

Copyright: ( 2015 Le Luyer, et al. This is an open-access article distributed unde the terms of the Creative Commons Attribution License, which permits unrestricted use, distribution, and reproduction in any medium, provided the original author and source are credited. 

Vertebrae: A Common Abnormality in Rainbow Trout (Oncorhynchus mykiss, Walbaum) Fed a Low-Phosphorus Diet. Next Generat Sequenc \& Applic 2: 112. doi:10.4172/2469-9853.1000112

and cell types, interconnected by intervertebral tissue and undergoing compression forces during swimming. As in other vertebrates, under non-pathological conditions, a tight balance in bone tissue is maintained between bone apposition by osteoblasts and bone resorption, resulting in a continuous turnover. In trout and in other species that belong to basal teleostean lineages, osteoblasts are trapped in the matrix and become osteocytes, a condition that defines cellular bone [21]. The osteocytes also play a role in bone apposition and bone resorption around their lacunae [22]. In vertebrae, chondrocytes are also present at the base of neural and hemal arches (basalia). Their progressive endochondral mineralization enables the complete fusion of the arches with the vertebral body during juvenile growth, at least in the caudal portion of the axial skeleton [9]. Thus, the occurrence of vertebral anomalies reflects a disturbance of the homeostatic mechanisms and well-coordinated actions of several cell types.

The molecular pathways involved in P-deficiency-induced deformities are far from being well understood. In Atlantic salmon (Salmo salar) presenting vertebral fusions [23,24], more than 20 actors including transcription factors and genes encoding molecules involved in bone cell activation and differentiation, and extracellular matrix (ECM) formation were detected. Similarly, a large-scale study on the impact of photoperiod on vertebral bone formation was conducted in Atlantic salmon [25] and highlighted the structural role of COL11a1 in the occurrence of vertebral deformities. Recently, characterization of vertebrae transcriptomes in sea bream (Sparus auratus) [26] and rainbow trout [27] suggested that homologs of key genes involved in bone metabolism are conserved in vertebrates. As bone metabolism in fish is complex, transcriptomic-wide analyses may be the best approach to identify the principal affected pathways during $\mathrm{P}$ deficiency.

A recent study [20] showed that the most common deformities observed in P-deficient all-female triploid rainbow trout are pronounced biconcave and homogeneously-compressed vertebrae, biconcave vertebrae being considered an early stage of vertebral compression. Most of the fish undergoing prolonged $\mathrm{P}$ deficiency develop vertebral abnormalities, however, some individuals are able to maintain a normal phenotype. Given this phenotypic plasticity, it is of interest to better understand the cellular mechanisms underlying the appearance of vertebral abnormalities.

The aim of this study was to identify molecular changes that are involved in the appearance of pronounced biconcave vertebrae (Figure 1) when trout are fed a low-P diet. We compared gene expression in normal and biconcave vertebrae from P-deficient all-female triploid rainbow trout using next-generation sequencing (NGS) on the Illumina HiSeq2000 platform. To support the transcriptomic results, in each

Normal
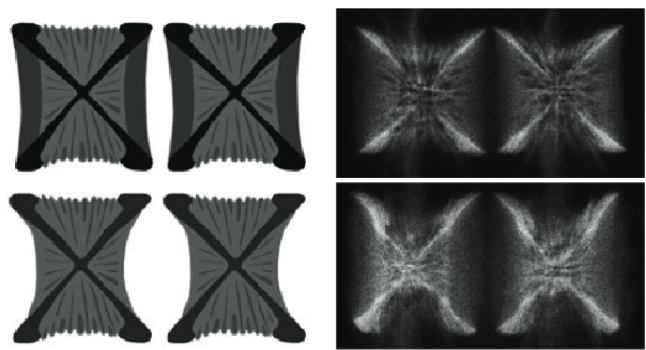

Figure 1: Illustrations (left) and X-rays (right) of the reported vertebral phenotypes in this study (normal and pronounced biconcave, respectively) and associated with mineral deficiency in teleost. Illustrations were modified after Witten et al. [28] For further details regarding the phenotypes see Poirier Stewart et al. [20]. individual we characterized the $\mathrm{P}$ status and the quality of bone tissue based on chemical, histological and biomechanical determinations. We discuss our findings with regard to transcription regulation and the formation, mineralization and resorption of bone tissue.

\section{Material and Methods}

\section{Husbandry}

All-female triploid rainbow trout $(\mathrm{N}=1,680$, initial mass 60.8 $\pm 1.6 \mathrm{~g}$ ) were transferred from St-Alexis-des-Monts Inc., Canada to experimental facilities at the Laboratoire de recherche des sciences aquatiques (LARSA), Université Laval in Québec City (Canada). An acclimation period of two weeks took place, during which fish were fed a commercial feed (Corey Optimum $3 \mathrm{~mm}$ ) in accordance with manufacturer's tables. Thereafter, fish $(\mathrm{N}=840)$ were fed with practical P-deficient diet (available P: 0.29\%) using either apparent satiation or pair-fed feeding regimes. The P-deficient diet was found to induce severe deficiency in rainbow trout as already reported $[16,19,20]$.

The fish were reared for 27 weeks in six circular tanks $(2000 \mathrm{~L})$ in a recirculating aquaculture system (6 replicates, $\mathrm{n}=140 / \operatorname{tank}, 8.5$ to $52.7 \mathrm{~kg} \mathrm{~m}^{-3}, 12 \pm 0.3^{\circ} \mathrm{C}$ ), with near oxygen saturation and at constant, long (LD, 18:6) photoperiod. During experimental manipulations, fish were anaesthetised in a MS-222 bath ( $75 \mathrm{mg} \mathrm{L}^{-1}$; Syndel International Inc., Vancouver, BC, Canada). Experiments took place in compliance to the guidelines of the Canadian Council on Animal Care (1984) and supervised by the Animal Protection Committee of Université Laval.

\section{Deformities assessment and tissue sampling}

At week 5, all trout were PIT-tagged with a microchip enabling individual survey (weight, length and condition factor: $\mathrm{K}=10^{5} \times \mathrm{g} /$ $\mathrm{mm}^{3}$ ) for each sample. X-rays permitted individual monitoring and was performed at week 5, 15 and 24. X-rays were performed on the trunk-caudal region of all fish at $60 \mathrm{kV}(2 \mathrm{~s}, 15 \mathrm{~mA}$, distance of 40 $\mathrm{cm})$. Monitoring of vertebral abnormalities was assessed directly on developed films and abnormalities along the vertebral axis were sorted according to the classification proposed for Atlantic salmon [28]. Fish were associated to a phenotype when all caudal vertebrae (V31-V44) showed a homogenous pattern of abnormalities (see details in [20]). At the termination of the study (week 27), P-deficient fish displaying normal ( $\mathrm{n}=3 \mathrm{fish}$ ) and pronounced biconcave ( $\mathrm{n}=3$ fish) vertebrae were randomly sampled. This abnormality type was chosen as it was by far the most represented type observed in the current study [20]. Moreover, pronounced biconcave vertebrae are related to early stages of mineral deficiency and might be a transition stage to vertebral compression, which are of major concern for fish growth [28]. For each fish, blood was collected from the caudal aorta, centrifuged, and the plasma stored at $-20 \mathrm{C}$. Scales were scraped from tail to head and were collected in a $70^{\circ}$ ethanol solution. Caudal vertebrae were removed for bone histology (V32-33 and V38-39), bone genomics (V35-37), bone structure (V4042), biomechanics and bone chemistry (V31-32 and V43-44). All of these analyses were conducted on vertebrae from the same individuals.

\section{Inorganic phosphorus in plasma}

Colorimetric assays (phosphomolybdate; K003912, JAS diagnostics, Inc., USA) were performed to measure inorganic phosphates $\left(\mathrm{Pi}=\mathrm{PO}_{4}^{3-}\right)$ in individual samples of plasma $(150 \mu \mathrm{l})$ using an automatic spectrophotometer (Trilogy Multi-purpose diagnostic analyzer system, Drew Scientific, USA).

\section{Bone histology}

To quantify the mineralization of basalias, two attached vertebrae (V38-39) were fixed in $4 \%$ formaldehyde and decalcified in $25 \%$ decal 
Citation: Le Luyer J, Deschamps MH, Proulx E, Poirier Stewart N, Droit A, et al. (2015) RNA-Seq Transcriptome Analysis of Pronounced Biconcave Vertebrae: A Common Abnormality in Rainbow Trout (Oncorhynchus mykiss, Walbaum) Fed a Low-Phosphorus Diet. Next Generat Sequenc \& Applic 2: 112. doi:10.4172/2469-9853.1000112

Page 3 of 13

solution for $24 \mathrm{hrs}$. Vertebrae were then dehydrated in graded series of ethanol prior to being embedded in paraffin. Up to 15 adjacent longitudinal cross-sections $(5 \mathrm{~m})$ from either side of the mid-region of the vertebrae (i.e., where the notochord canal is present at the narrowest) were obtained using a microtome (Microm HM 330, Heidelberg, Germany). The sections were stained using Sirius red techniques [29]. Two other attached vertebrae V33-34 (fixed in cold acetone) were embedded in glycol methacrylate. One longitudinal section was obtained by sanding one side of the block to the mid-region of the vertebrae and by cutting the other side of the block to a thickness of $1.5 \mathrm{~mm}$ using a band saw and a cutting guide. The sections were polished before being stained using Von Kossa techniques [30].

Morphometrical results at the vertebral body endplates (VBE) were estimated from the Von Kossa sections. For each individual, tiff images from the longitudinal section (4x magnification) were obtained using a digital camera (EXI Blue Q35618, Q Imaging Inc., BC, Canada) mounted on a microscope (Nikon eclipse E600) and captured with Q-capture Pro 7 software (Q Imaging Inc., BC, Canada). Once transferred to ImageJ v1.40 (U. S. National Institutes of Health, USA, http://rsb.info.nih. gov./ij/) software (with Java, 32 bits), the freehand selection tool was used to manually delineate (graphic tablet Intruos3, model PTZ-631W, Wacom Co. Ltd, China) areas and calculate a number of pixels for the mineralized trabecular area $(\mathrm{tb})$ and total bone area, comprising tb and the un-mineralized osteoid at the apposition front (os), for each vertebral body endplate (VBE) ( $\mathrm{n}=8$ /individual). The mean proportion of os (\%) at the VBE was calculated as follows: os/(os + tb) x 100. The length $\left(\mathrm{L}_{\mathrm{VBE}}\right)$ and thickness $\left(\mathrm{T}_{\mathrm{VBE}}\right)$ of the vertebral body endplates were also measured. $\mathrm{L}_{\mathrm{VBE}}$ included the total distance from the notochord canal to the apposition front. $\mathrm{T}_{\mathrm{VBE}}$ was defined as the perpendicular distance from the point of attachment of the notochord tissue to the inner limit of the compact bone layer. A schematic representation including the different vertebral body parts mentioned in this section is proposed in Figure 2.

The relative mineralization of the basalias was estimated from digital pictures of Sirius red sections at 200x magnification $(\mathrm{N}=5$ images/basalia/individual section) using a digital camera (Infinity23C; Lumenera 7.0.3.23) mounted on a binocular microscope (Olympus BX53) and captured with Image-Pro Plus software (version 7.0.0.591). The basalias were cut and cell nuclei removed using the selection tools

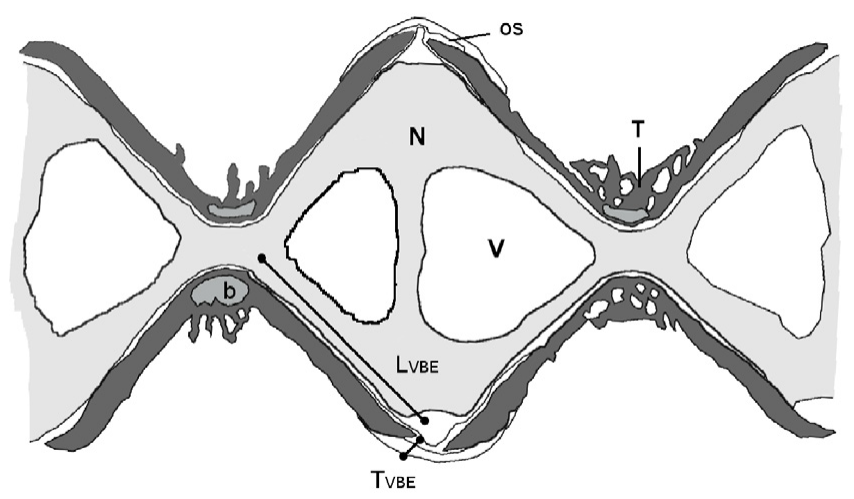

Figure 2: Scheme of a longitudinal section of the mid-region of two adjacent vertebrae including basalias and notochordal tissues. With $b=$ basalia; $L_{\text {}}$ = length of the vertebral body endplate; $\mathrm{N}=$ notochordal tissue; os = osteoid; $\mathrm{T}=$ trabecular bone; $\mathrm{V}=$ vacuolated tissue; $\mathrm{VBE}$ = vertebral body endplates; $\mathrm{T}_{\mathrm{VBE}}=$ thickness of the vertebral body endplate.

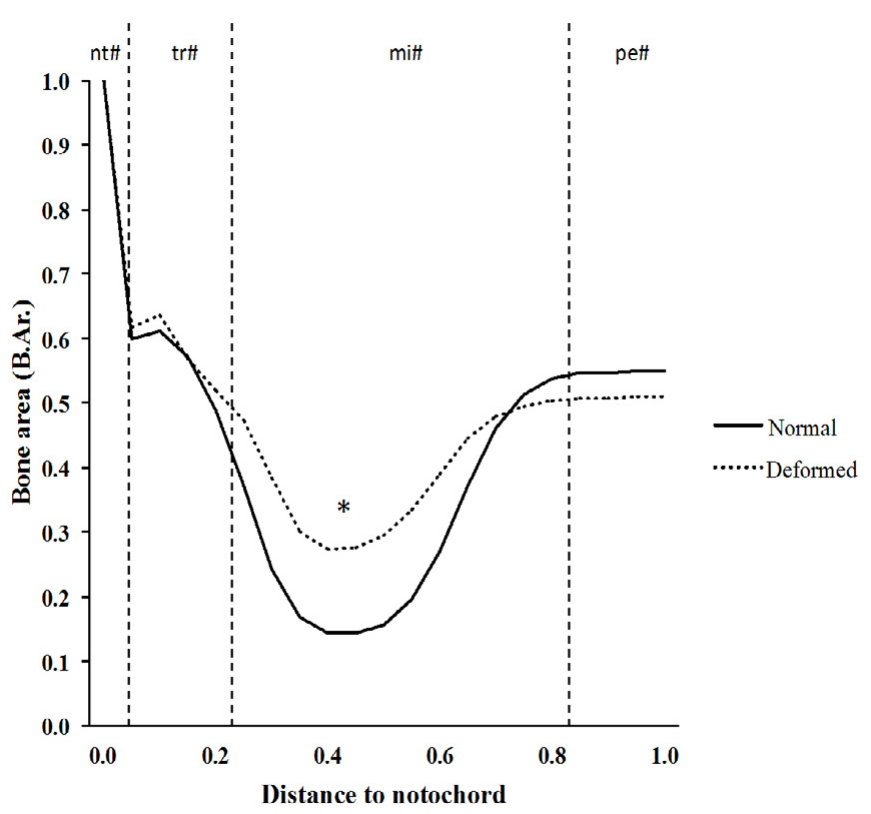

Figure 3: Vertebral bone profile for normal and deformed groups. Bone area (expressed as a ratio from 0 to 1 ) as a function of the relative distance to the notochord $. \mathrm{nt}=$ notochord $\mathrm{area} ; \mathrm{tr}=$ transition area; $\mathrm{mi}=$ middle area; pe $=$ periphery area. Asterisk $\left(^{*}\right)$ shows a slight significant difference for the lost of bone in the middle area between groups (ANOVA $\mathrm{P}<0.05 ; \mathrm{n}=3 \mathrm{fish} /$ condition).

of GIMP v2.8.10 software (ww.gimp.org). The number of pixels for two RGB colours (unmineralized collagen $=$ red $(222,333,136)$; mineralized collagen=grey $(142,59,142)$ were analysed using ImageJ and colour deconvolution Plug-In as published elsewhere [31]. The proportion of mineralized and unmineralized areas of the basalias were calculated as the number of pixels for a specific colour divided by the total number of all defined pixels $\times 100$.

\section{Bone profiling}

Bone density and structure of vertebrae V40-42 were estimated using the approach of the modelling of bone area profiles according to previously-published methods [32] and presented in Figure 3. Briefly, total bone area (Tt-B.Ar.) and mean bone area according to the relative distance from the notochordal canal were measured using Bone profiler software [33] and modeled using 12 parameters in four predefined regions of the vertebral body (notochord, transition, middle and periphery). Measurements were performed on $125 \mu \mathrm{m}$-thick transverse sections of the mid-region of the vertebrae, in which the notochord canal is the narrowest and bone tissue the largest. Virtual cross-sections were obtained from $\mu \mathrm{CT}$ images (National Museum of Natural History, France).

\section{Biomechanics}

Four vertebrae (V31, 32, 43 and 44) were used for mechanical testing prior mineral content analyses. Neural and haemal arches were removed, and the amphicoelus centra were compressed along the cranial-caudal axis at a steady rate of deformation $\left(0.1 \mathrm{~mm} \mathrm{sec}^{-1}\right)$ using a texture analyzer (TA-XT2 Texture Analyzer, Stable Micro Systems, UK) and the Texture Expert Exponent 32 acquisition software (TE32 version 5.1.1.0, Texture Technologies, USA). For each vertebra, load $(\mathrm{N})$ and deformation $(\mathrm{mm})$ data were continuously recorded and the stiffness (g.mm $\mathrm{mm}^{-1}$ was calculated according previously-published methods [34]. 

Vertebrae: A Common Abnormality in Rainbow Trout (Oncorhynchus mykiss, Walbaum) Fed a Low-Phosphorus Diet. Next Generat Sequenc \& Applic 2: 112. doi:10.4172/2469-9853.1000112

\section{Mineralization status}

The mineral content was determined for scales and vertebrae (V31, $32,43$ and 44$)$. The samples were dehydrated in a graded series of ethanol $\left(70^{\circ}, 90^{\circ}, 100^{\circ} ; 24 \mathrm{~h} / \mathrm{bath}\right)$, and lipids were extracted in acetone (two baths of $24 \mathrm{~h}$ ), then in trichloroethylene (two baths of $24 \mathrm{~h}$ ). Diets and tissues were analyzed for dry matter (drying in a vacuum oven for $18 \mathrm{~h}$ at $105^{\circ} \mathrm{C}$ ) and ash (incineration in a muffle furnace for $18 \mathrm{~h}$ at $550^{\circ} \mathrm{C}$ ), weighed to the nearest $0.1 \mathrm{mg}$ according to AOAC guidelines (1990). P content was determined by ion chromatography (ICS-3000, Dionex Corporation, Sunnyvale, CA) following ash digestion in nitric acid solution and filtering (Whatman paper No. 1, rinsed 3 times). Separation was carried out on an IonPac AS 17 column (Dionex) with an IonPac AG 17 guard column. For sample loading, a $50 \mu \mathrm{l}$ sample loop (PEEK) was used. Multi-step gradient concentrations ranging from 9 to $30 \mathrm{mM}$ allowed for the complete separation of phosphorus anions in aqueous media in $16 \mathrm{~min}$ at a constant flow rate of $1.0 \mathrm{ml} . \mathrm{min}-1$.

\section{Sequencing}

Three connected vertebrae (V35-37, comprised of ligaments and intervertebral tissue) were rapidly dissected, arches removed and the remaining vertebral body quickly cleaned of flesh by rinsing and brushing with PBS (1X) to remove all muscle tissue. The vertebrae from each individual was then immersed in liquid nitrogen, crushed into a fine powder, and total RNA extracted using TRIZOL Reagent (Life Technologies) at a ratio of $1 \mathrm{ml}$ per $100 \mathrm{mg}$ tissue according to manufacturer's recommendations. Total RNA was subjected to DNAse treatment on a column (ArcturusPicoPure RNA isolation kit, Life Technologies), and integrity and purity assessed using a Bioanalyzer 2100 (Agilent Technologies). RNA was also quantified using a NanoDrop ND-1000 spectrophotometer (NanoDrop Technologies Inc.). For each sample, $1 \mu \mathrm{g}$ of total RNA was used to create cDNA libraries with TrueSeq Sample Prep kit v2 (Illumina, San Diego, CA, USA) following manufacturer's instructions. Resulting libraries were quantified using a Bioanalyzer 2100 (Agilent Technologies). Samples were multiplexed (6 samples per lane) and sequenced at McGill genomic platform with HiSeq2000 sequencer and 100 paired-end (PE) technology.

\section{Differentially expression study and annotation}

Reads from HiSeq2000 Illumina were first processed with Trimmomatic v0.30 [35] to remove low quality (trailing: 20, lowest quality: 30$)$ and short reads ( $<60 \mathrm{bp}$ ). Trimming with Trimmomatic also included removal of Illumina adapters together with the most common contamination vectors from UniVec database (https://www. ncbi.nlm.nih.gov/tools/vecscreen/univec/). Read abundance and differentially-expressed genes were estimated using Trinity pipeline published recently for non-model species [36] by mapping reads on a reference transcriptome for rainbow trout bone tissue [27]. Briefly, gene abundance estimations have been assessed individually using RSEM tools [37] and bowtie v1.0.0 [38]. The differentially-expressed gene assessment based on RPKM values, was conducted using both EdgeR v3.8.5 [39] and DESeq v1.18.0 [40] packages with correction of false discovery rate (FDR) of $5 \%$. Only common genes detected as differentially expressed with both analyses have been used for further analyses and functional annotation. Blast-x searches (cut-off e-value $<10-8$ ) were performed on Uniprot-Swissprot database. Gene ontology (GO) association was realised using GOminer software [41]. To estimate the proportion of long non-coding RNAs (lncRNAs), remaining sequences non-matching against Uniprot database were analyzed using PLEK software [42] (length threshold $>200 \mathrm{bp}$ ).
Visualization of biological functions was conducted using IPA core analysis (Ingenuity Systems, www.ingenuity.com). The Ingenuity platform allows novel protein sequence association with networks, canonical pathways and biological functions relative to human and mouse systems. The genes specifically related to bone metabolism were manually searched by compiling relevant metabolic pathways (Table 1). The analysis pipeline for gene expression quantification is described in Figure 4.

\section{RT-qPCR}

We used RT-qPCR to assess differential gene expression for keys genes involved in each cellular types: MEF2C, TRAP and BGLAP, for chondrocytes, osteoclasts and osteoblasts, respectively. For each sample, total RNA was converted into complementary DNA (cDNA) using Omniscript reverse transcriptase kit (Qiagen). Specific primers (Table 2) were designed using Primer Quest from DNA Technologies (IDT, Coralville, IA) for Real-Time quantitative PCR (480 Roche lightcycler 2.0, Roche Diagnostics, Canada) and SYBR green I staining [43]. Quality and specificity of amplicons were visually confirmed on $2 \%$ gel electrophoresis and by DNA sequencing. A normalization factor for each sample was assessed using GeNorm software [44] based on two housekeeping gene expression levels, $\beta$-actin and Heat Shock Protein 90B (HSP90B). Expression of genes assessed by RT-PCR is presented in Figure 5.

\section{Statistical analysis}

The histological results and fish performance are reported as mean \pm standard error of the mean (sem). After ensuring normality (Shapiro-Wilk, Skewness and Kurtosis tests) and homoscedasticity (Bartlett test), data were analyzed using one-way ANOVAs. For the length of trabecular bone observed with Von Kossa, data were Log10 transformed. The results were assumed to be significant at $p$-values below 0.05 . All the tests were conducted with $\mathrm{R}$ software version 3.0.2 [45] using Moments package [46].

\section{Results and Discussion}

This experiment employed all-female triploid individuals fed with a single P-deficient diet in order to overcome possible factors increasing phenotypic variation such as differences in somatic growth, sex and sexual maturation. After 27 weeks of feeding low-P diets, mortality was similar and negligible (3.2\%) in all tanks and no significant difference in growth performance was observed in control and experimental fish. Moreover, no difference in length, weight and condition factor (Table 3 ) were observed between experimental individuals ( $\mathrm{p}=0.06$ and 0.09 , respectively) ([20]) and external visualisation could not detect any defects of conformation (gross deformity).

Here, we focused on pronounced biconcave phenotype because of its high frequency in salmonids prone to mineral deprivation and because it is considered as an initial development stage of more severe compression deformities [20,28]. Pronounced biconcave vertebrae after 27 weeks were observed in $24.9 \%(\mathrm{~N}=113)$ of the fish. Only $5.5 \%$ $(\mathrm{N}=25)$ of individuals displayed normal vertebrae (healthy control fish) all along experiments (i.e., from week 9 to week 27). Other fish showing normal phenotype at week $27(31.1 \% ; \mathrm{N}=141)$ were not considered because they were known to recover from an abnormal phenotype at week 18. Moreover, other types of deformities such as homogeneous compressions (29.4\%; $\mathrm{N}=133$ ), small and widely spaced vertebrae $(5.5 \% ; \mathrm{N}=25)$ and other types of deformities $(3.5 \% ; \mathrm{N}=16)$ were also observed but were not considered for this study. 
Citation: Le Luyer J, Deschamps MH, Proulx E, Poirier Stewart N, Droit A, et al. (2015) RNA-Seq Transcriptome Analysis of Pronounced Biconcave Vertebrae: A Common Abnormality in Rainbow Trout (Oncorhynchus mykiss, Walbaum) Fed a Low-Phosphorus Diet. Next Generat Sequenc \& Applic 2: 112. doi:10.4172/2469-9853.1000112

Page 5 of 13

\begin{tabular}{|c|c|c|c|c|}
\hline Gene header & Uniprot Acc. & Gene name & $\log 2 \mathrm{FC}$ & FC \\
\hline \multicolumn{5}{|c|}{ Down-regulated genes in deformed vertebra } \\
\hline comp149051_c0 & A4IFW2 & Protein tyrosine phosphatase receptor type $\mathrm{F}$ & 1.64 & 3.12 \\
\hline comp138459_c1 & Q64487 & Protein tyrosine phosphatase receptor type D & 1.62 & 3.08 \\
\hline comp145566_c4 & Q96JQ0 & Dachsous Cadherin-Related 1 & 1.55 & 2.93 \\
\hline comp144178_c2 & Q6ZRS2 & Snf2-related CREBBP activator protein & 1.49 & 2.8 \\
\hline comp146067_c0 & P35556 & Fibrillin 2 & 1.42 & 2.67 \\
\hline comp132718_c0 & P45481 & CREB binding protein & 1.38 & 2.61 \\
\hline comp149014_c5 & Q8BNA6 & FAT atypical cadherin 3 & 1.37 & 2.59 \\
\hline comp144153_c0 & 095644 & Nuclear factor of activated T-cells, calcineurin-dependent & 1.31 & 2.49 \\
\hline comp133481_c0 & Q9Y3S1 & Serine/threonine-protein kinase WNK2 & 1.31 & 2.49 \\
\hline comp148107_c0 & Q8WYB5 & K (Lysine) acetyltranferase 6B & 1.24 & 2.35 \\
\hline comp147565_c0 & Q5I598 & Methylenetetrahydrofolate (NAD(P)H) & 1.22 & 2.33 \\
\hline comp143062_c0 & Q13332 & Protein tyrosine phosphatase receptor type S & 1.19 & 2.28 \\
\hline comp126856_c0 & P17948 & Vascular endothelial growth factor receptor 1 & 1.17 & 2.24 \\
\hline comp134803_c0 & Q14517 & FAT atypical cadherin 1 & 1.14 & 2.21 \\
\hline comp143303_c7 & Q8VI24 & SATB homeobox 2 & 1.12 & 2.18 \\
\hline comp145538_c0 & Q99715 & Collagen type $\mathrm{X} 2$ alpha 1 & 1.12 & 2.18 \\
\hline comp131699_c0 & F1QCC6 & Glypican 1 & 1.1 & 2.14 \\
\hline comp146160_c1 & Q9Z180 & SET binding protein 1 & 1.1 & 2.14 \\
\hline comp149571_c0 & Q9P2D1 & Chromodomain helicase DNA binding protein & 1.09 & 2.13 \\
\hline comp140581_c1 & Q92794 & K (Lysine) acetyltranferase $6 \mathrm{~A}$ & 1.05 & 2.07 \\
\hline comp128868_c0 & O75581 & Low density lipoprotein receptor-related protein 6 & 1.04 & 2.05 \\
\hline comp142199_c3 & 070496 & Chloride channel voltage-sesitive 7 & 1.01 & 2.01 \\
\hline comp147156_c2 & O89026 & Roundabout axon guidance receptor homolog 1 & 0.97 & 1.96 \\
\hline comp135768_c0 & P27658 & Collagen type VIII Alpha 1 & 0.97 & 1.97 \\
\hline comp149750_c0 & Q6V017 & FAT atypical cadherin 4 & 0.97 & 1.96 \\
\hline comp134251_c0 & Q8BTM8 & Filamin A, alpha & 0.97 & 1.96 \\
\hline comp141372_c4 & P98161 & Polycystin 1 & 0.95 & 1.93 \\
\hline comp144389_c0 & A7XYQ1 & Jackson circler protein 1 & 0.94 & 1.92 \\
\hline comp67973_c1 & Q6NSM8 & SIK family kinase 3 & 0.93 & 1.91 \\
\hline comp135751_c0 & Q9UBS9 & Sun domain-containing ossification factor & 0.93 & 1.9 \\
\hline comp135751_c0 & Q9QYP0 & Multiple EGF-like-domain 8 & 0.91 & 1.88 \\
\hline comp145151_c0 & P26012 & Integrin $\beta 8$ & 0.9 & 1.87 \\
\hline comp136915_c0 & Q13233 & Mitogen-activated protein kinase kinase kinase 1 & 0.89 & 1.85 \\
\hline comp149523_c0 & 088491 & Nuclear receptor binding SET domain protein 1 & 0.87 & 1.83 \\
\hline comp149485_c0 & Q9UHF7 & Trichorhinophalangeal syndrome I & 0.87 & 1.83 \\
\hline comp139497_c0 & P21359 & Neurofibromin 1 & 0.86 & 1.81 \\
\hline comp149389_c0 & O35607 & Bone morphogenetic protein receptor, type II & 0.85 & 1.81 \\
\hline comp146007_c0 & Q14938 & Nuclear factor 1/X (CCAAT-binding transcription factor) & 0.85 & 1.8 \\
\hline comp146819_c2 & Q90413 & Fibroblast growth factor receptor 4 & 0.81 & 1.75 \\
\hline comp146502_c4 & P20909 & Collagen type XI alpha 1 & 0.8 & 1.74 \\
\hline comp149588_c0 & A2A884 & Human immunodeficient virus type 1 enhancer binding protein 3 & 0.79 & 1.73 \\
\hline comp147279_c4 & P98160 & Heparan sulfate proteoglycan 2 & 0.78 & 1.72 \\
\hline comp144268_c0 & Q09472 & E1A binding protein $\mathrm{P} 300$ & 0.78 & 1.71 \\
\hline comp149387_c2 & Q8IXJ9 & Additional sex combs like 1 & 0.78 & 1.71 \\
\hline comp148958_c0 & Q9ULT8 & HECT domain containing E3 ubiquitin protein ligase 1 & 0.78 & 1.71 \\
\hline comp149573_c0 & P10587 & Myosin heavy chain 11 smooth muscle & 0.77 & 1.71 \\
\hline comp149223_c0 & P79114 & Unconventional myosin- $\mathrm{X}$, role $\mathrm{n}$ podosom belt osteoclast & 0.77 & 1.7 \\
\hline comp149584_c1 & P35580 & Myosin 10 & 0.75 & 1.68 \\
\hline comp138258_c6 & Q07889 & Son of sevenless homolog 1 & 0.69 & 1.61 \\
\hline comp147381_c0 & Q91YM2 & Rho GTPase activating protein 35 & 0.68 & 1.6 \\
\hline \multicolumn{5}{|c|}{ Up-regulated genes in deformed vertebra } \\
\hline comp146739_c2 & Q9YHV3 & Noggin-3 & -3.19 & 9.13 \\
\hline comp142082_c0 & P57102 & Heart and neural crest derivatives expressed 2 & -1.84 & 3.59 \\
\hline comp148652_c4 & Q9JK00 & Sodium channel voltage gated type III $\beta$ subunit & -1.63 & 3.09 \\
\hline comp149418_c1 & P05549 & Activating Enhancer-Binding Protein 2-Alpha & -1.51 & 2.84 \\
\hline
\end{tabular}


Citation: Le Luyer J, Deschamps MH, Proulx E, Poirier Stewart N, Droit A, et al. (2015) RNA-Seq Transcriptome Analysis of Pronounced Biconcave Vertebrae: A Common Abnormality in Rainbow Trout (Oncorhynchus mykiss, Walbaum) Fed a Low-Phosphorus Diet. Next Generat Sequenc \& Applic 2: 112. doi:10.4172/2469-9853.1000112

Page 6 of 13

\begin{tabular}{|c|c|c|c|c|}
\hline comp142563_c0 & P09238 & Matrix metallopeptidase 10 & -1.46 & 2.76 \\
\hline comp88970_c0 & P79703 & Jun B proto-oncogene & -1.42 & 2.67 \\
\hline comp144447_c2 & Q05826 & CCAAT/Enhancer binding protein (C/EBP) B & -1.27 & 2.42 \\
\hline comp135507_c0 & Q920D2 & Dihydrofolate reductase & -1.26 & 2.4 \\
\hline comp146593_c0 & P40148 & Osteocalcin & -1.13 & 2.19 \\
\hline comp144817_c0 & O75911 & Dehydrogenase/Reductase (SDR family member 3) & -0.93 & 1.9 \\
\hline comp121895_c0 & Q800Y2 & Matrix Gla protein & -0.86 & 1.82 \\
\hline comp136344_c0 & P52909 & Jun D proto-oncogene & -0.85 & 1.8 \\
\hline comp148539_c0 & P70061 & NK3 homeobox 2 & -0.85 & 1.81 \\
\hline comp147786_c0 & Q92008 & Sonic hedgehog & -0.84 & 1.79 \\
\hline comp143712_c0 & P25942 & CD40 molecule TNF receptor family member 5 & -0.78 & 1.72 \\
\hline comp149526_c0 & Q9NRR1 & Cytokine-Like 1 & -0.78 & 1.71 \\
\hline comp141960_c0 & P97831 & Twist 2 & -0.75 & 1.69 \\
\hline comp98891_c0 & 055052 & Macrophage migration inhibitory factor & -0.7 & 1.62 \\
\hline comp148620_c0 & P18203 & FK506 binding protein $1 \mathrm{~A}, 12 \mathrm{kDa}$ & -0.7 & 1.63 \\
\hline
\end{tabular}

Table 1: Selection of differentially-expressed genes putatively related to bone metabolism. The homologies were revealed with blast-x search against Uniprot-Swissprot database (cut-off evalue $<1 \mathrm{e}-8$ ). Differentially expressed genes were conserved if they display a unique and FDR $<5 \%$.

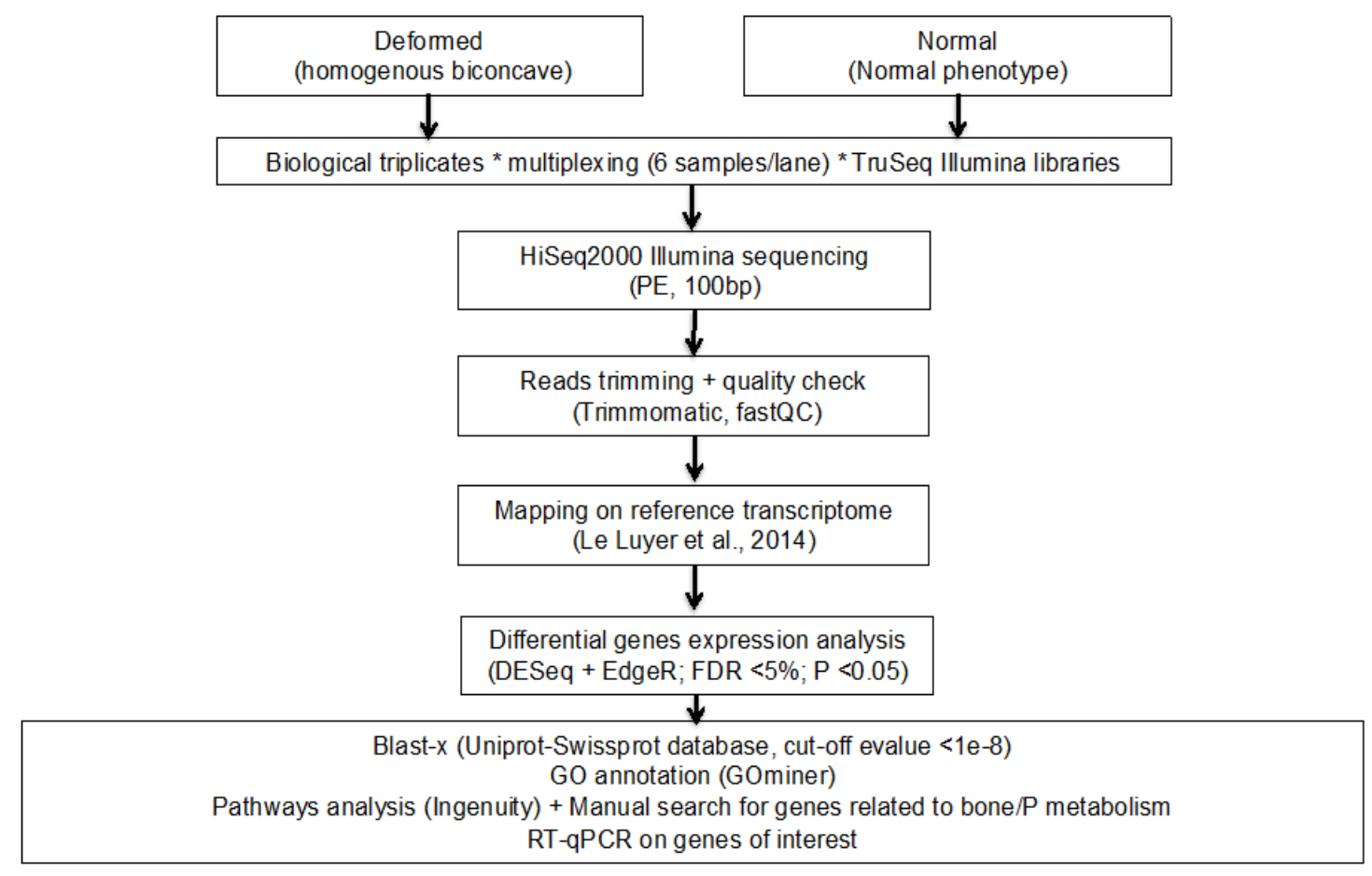

Figure 4: Pipeline of bioinformatic and gene expression analyses. PE=paired-ends.

\begin{tabular}{|c|c|c|c|c|c|}
\hline Gene name & Amplicon (bp) & Sense & Sequence (5'to3') & Length (bp) & $\mathrm{Tm}$ \\
\hline \multirow[t]{2}{*}{ TRAP } & \multirow[t]{2}{*}{162} & $\mathrm{~F}$ & CGGAGAGGTAACCAAGAACAAG & 22 & 60.2 \\
\hline & & $\mathrm{R}$ & TGTGACCGTCTATTTCGTCATC & 22 & 60 \\
\hline \multirow[t]{2}{*}{ Osteocalcin } & \multirow[t]{2}{*}{119} & $\mathrm{~F}$ & TCTCTCGСТСТСССТСТАACAC & 22 & 60.2 \\
\hline & & $\mathrm{R}$ & TCTATTCTGCAAGGCTTTTTGG & 22 & 60.6 \\
\hline \multirow[t]{2}{*}{ Mef2c } & \multirow[t]{2}{*}{158} & $\mathrm{~F}$ & ACCCACAAATACACAACACAGG & 22 & 59.7 \\
\hline & & $\mathrm{R}$ & GTCTGAAATGGAACCCTATTGC & 22 & 59.8 \\
\hline \multirow[t]{2}{*}{$\beta$-actin } & \multirow[t]{2}{*}{133} & $\mathrm{~F}$ & GAAAGACAGCTACGTGGGAGAC & 22 & 60.2 \\
\hline & & $\mathrm{R}$ & CTCGTTGTAGAAGGTGTGATGC & 22 & 59.8 \\
\hline \multirow[t]{2}{*}{ Heat shock protein $90 \mathrm{~B}$} & \multirow[t]{2}{*}{232} & $\mathrm{~F}$ & CGACTTCATCAAGCTGTTTGTC & 22 & 59.9 \\
\hline & & $\mathrm{R}$ & CTTGTCGTTGTACTGCTCTTCG & 22 & 60.1 \\
\hline
\end{tabular}

Table 2: Primer sequences, product sizes and annealing temperature (Tm) of selected genes for RT-qPCR. 

Vertebrae: A Common Abnormality in Rainbow Trout (Oncorhynchus mykiss, Walbaum) Fed a Low-Phosphorus Diet. Next Generat Sequenc \& Applic 2: 112. doi:10.4172/2469-9853.1000112
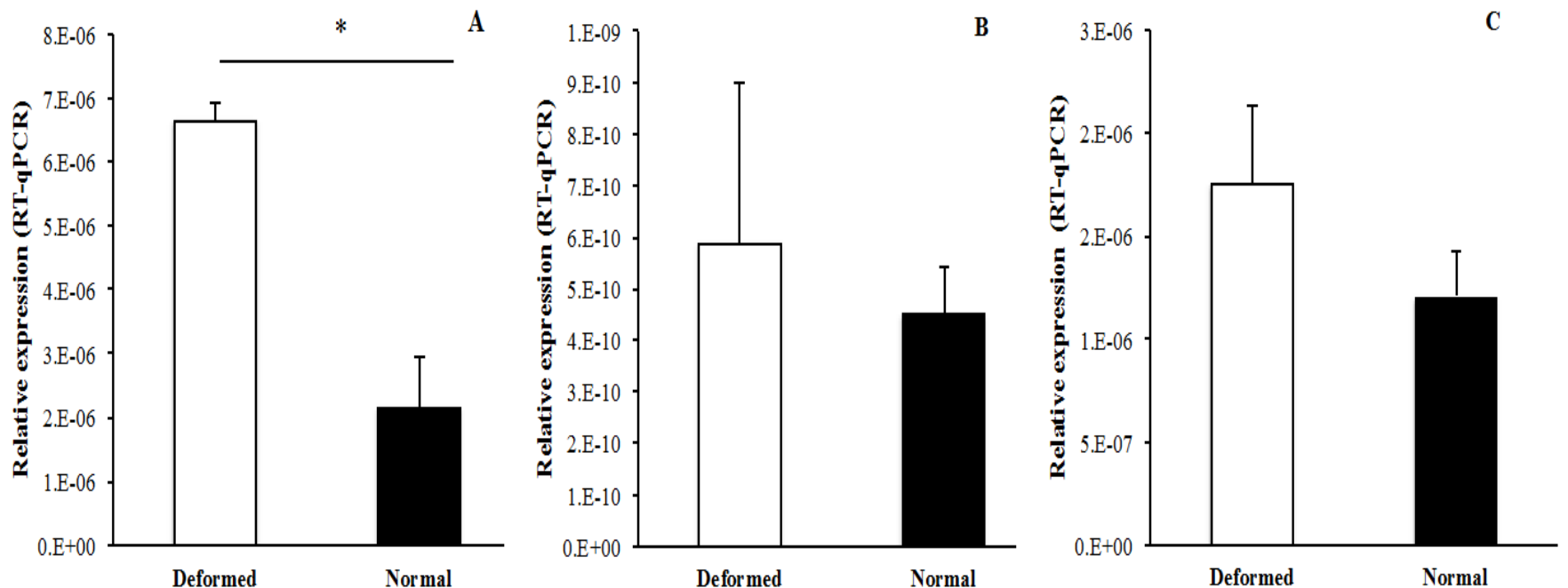

Figure 5: RT-PCR results on selected bone-related genes. Graphs represent the gene expression analyses performed independently by RT-qPCR with A) BGLAP, B) MEF2C and C) TRAP. Asterisk $\left(^{*}\right)$ represent significant difference between groups (ANOVA P $\left.<0.05\right)$. Data represent means $(n=3$ fish) \pm sem.

\begin{tabular}{|c|c|c|c|c|c|c|c|c|c|c|c|}
\hline \multirow[b]{2}{*}{ Phenotypes } & \multicolumn{5}{|c|}{ Sirius red (basalias) } & \multicolumn{5}{|c|}{ Von Kossa (trabecular bone) } & \multirow{2}{*}{$\begin{array}{c}\mu \mathrm{XT} \\
\text { Tt.B.Ar } \\
(\%)\end{array}$} \\
\hline & \multicolumn{2}{|c|}{$\begin{array}{c}\text { Non mineralized } \\
\text { area }(\%)\end{array}$} & \multicolumn{2}{|c|}{$\begin{array}{c}\text { Intermediary } \\
\text { mineralized } \\
\text { area }(\%)\end{array}$} & $\begin{array}{c}\text { Mineralized area } \\
(\%)\end{array}$ & $\begin{array}{l}\text { Total area } \\
\left(\mu \mathrm{m}^{2}\right)\end{array}$ & \multicolumn{2}{|c|}{$\begin{array}{l}\text { Mineralized area } \\
\qquad\left(\mu \mathrm{m}^{2}\right)\end{array}$} & \multicolumn{2}{|c|}{ Osteoid $\left(\mu \mathrm{m}^{2}\right)$} & \\
\hline Normal & \multicolumn{2}{|c|}{$59.4 \pm 11.5$} & \multicolumn{2}{|c|}{$32.0 \pm 9.6$} & $8.5 \pm 1.9$ & $5931.2 \pm 249.8$ & \multicolumn{2}{|c|}{$5711.0 \pm 233.1$} & \multicolumn{2}{|c|}{$220.1 \pm 16.7$} & $39.3 \pm 3.2$ \\
\hline Deformed & \multicolumn{2}{|c|}{$68.6 \pm 7.8$} & \multicolumn{2}{|c|}{$17.7 \pm 2.0$} & $13.7 \pm 6.0$ & $5442.0 \pm 110.1$ & \multicolumn{2}{|c|}{$5166.1 \pm 99.8$} & \multicolumn{2}{|c|}{$275.9 \pm 10.7$} & $43.3 \pm 3.6$ \\
\hline \multirow{2}{*}{ Phenotypes } & \multirow{2}{*}{$\begin{array}{l}\text { Fork length } \\
\quad(\mathrm{cm})\end{array}$} & \multicolumn{2}{|c|}{ Weight } & Condition & Stiff. & P Vt. & Ash Vt. & \multicolumn{2}{|c|}{ P Sc. } & Ash Sc. & P plasma \\
\hline & & \multicolumn{2}{|c|}{ (g) } & factor (K) & $(\mathrm{N} / \mathrm{mm})$ & $(\%)$ & $(\%)$ & \multicolumn{2}{|c|}{$(\%)$} & (\%) & (mg/dl) \\
\hline Normal & $35.1 \pm 0.6$ & \multicolumn{2}{|c|}{$657.3 \pm 33.2$} & $1.5 \pm 0.0$ & $140.2 \pm 6.3^{*}$ & $9.3 \pm 0.3$ & $59.4 \pm 0.5^{*}$ & \multicolumn{2}{|c|}{$5.5 \pm 0.1$} & $29.8 \pm 0.7^{*}$ & $11.5 \pm 0.4^{*}$ \\
\hline Deformed & $32.6 \pm 0.8$ & \multicolumn{2}{|c|}{$526.9 \pm 49.6$} & $1,6 \pm$ & $80.3 \pm 9.0^{*}$ & $8.8 \pm 0.3$ & $55.0 \pm 0.4^{*}$ & 4.4 & & $26.3 \pm 1$ & $7.6 \pm 0.7^{*}$ \\
\hline
\end{tabular}

Table 3: Growth, condition factors, stiffness, $P$ mineralization status (dry tissues) calculated as ash or $P$ content and results of histological observations by Sirius red and Von Kossa. Values are expressed as means $(n=3) \pm$ sem. P: Phosphorus; Sc: Scales; Vt: Vertebrae; Tt.B.Ar: Total Bone Area. Asterisks $\left({ }^{*}\right)$ represent significant difference $(\mathrm{P}<0.05)$.

The $\mathrm{P}$ status of deformed fish displaying pronounced biconcave vertebra was negatively affected as shown by the lower ash content in their scales and vertebra, and the lower plasma Pi concentration (Table 3). Our results are consistent with previous observations that showed a significant correlation between low mineral content in scales and higher occurrence of vertebral deformities in rainbow trout fed low-P diets [19]. Whether the altered mineral status resulted from differences in individual $\mathrm{P}$ requirements or from difference in feed intake due to limited food supply inherent to feeding approaches and/ or competition between congeners deserves to be further studied. The lower mineralization of biconcave vertebrae resulted in lower biomechanical competence when submitted to a compression force (stiffness, Table 3), a finding which is coherent with similar results reported in Atlantic salmon $[47,48]$. Lower P content and mechanical strength are recognised as initial indicators of deformity occurrence, particularly for the various compression types [48]. Phenotypically, pronounced biconcave vertebrae differ from normal vertebrae by their apparent "transparent" vertebral body endplates upon X-ray evaluation (Figure 1); the shape of the vertebral body appears as a vertical apple core and the X-shaped centrum of the vertebrae display more acute angles $[20,28]$. Therefore, our results may suggest that the lower biomechanical competence of the vertebrae promotes the alteration of the vertebral body endplates (apposition site of newly formed bone) as well as in binding the $\mathrm{X}$-structure of the vertebral body (more acute angles). If this hypothesis is true, the mechanisms of vertebral compression would not only affect intervertebral tissues at the vertebral body endplates as suggested by some authors $[23,28]$, but also the entire structure of the vertebral body.

A recent study from our group reported the first reference transcriptome for rainbow trout vertebral bone and its characterization revealed that key regulators of bone metabolism have been well conserved in vertebrates and are present in trout [27]. Here, we compared the overall gene expression by mapping paired-end reads on the transcriptomes in pronounced biconcave (deformed) vertebra with normal vertebral phenotype after 27 weeks of feeding a P deficient diet (Table 4). Using edgeR and DESeq package (FDR $<5 \%$; $<0.05)$, a total of 1289 genes were found differentially expressed. Of these, 700 were down- and 589 up-regulated in biconcave vertebrae. A blast-x search (cut-off 1e-8) was conducted to identify these sequences in Uniprot-Swissprot database reference and only genes with unique match were conserved. A total of 793 sequences $(61.5 \%$ of the total genes) corresponding to 485 down- (61.9\%) and 308 up-regulated (38.8\%) genes were associated with known proteins. Furthermore, from the 1289 genes $\mathrm{DE}, 23.1 \%(\mathrm{~N}=298)$ were associated with putative lncRNAs. The unidentified sequences were not retained in our analyses 

Vertebrae: A Common Abnormality in Rainbow Trout (Oncorhynchus mykiss, Walbaum) Fed a Low-Phosphorus Diet. Next Generat Sequenc \& Applic 2: 112. doi:10.4172/2469-9853.1000112

\begin{tabular}{|l|c|c|}
\hline Sequencing & Normal & Deformed \\
\hline Ave. number reads & $44.6 \mathrm{M}$ & $46.1 \mathrm{M}$ \\
\hline Ave. number trimmed reads & $35.3 \mathrm{M}$ & $37.8 \mathrm{M}$ \\
\hline Ave. mapping (\%) & 71.7 & 71.6 \\
\hline DE analysis & \multicolumn{2}{|c|}{ Def. Vs. Norm. } \\
\hline Up-regulated in Def. & \multicolumn{2}{|c|}{700} \\
\hline Down-regulated in Def. & \multicolumn{2}{|c|}{589} \\
\hline Total & \multicolumn{2}{|c|}{1289} \\
\hline Match Uniprot & \multicolumn{2}{|c|}{793} \\
\hline
\end{tabular}

FDR: $5 \%$, triplicates. Unique gene match in Uniprot-Swissprot database (cut-off evalue $<1 \mathrm{e}-8)$. Analyses on genes levels expression with both edgeR and DESeq packages.

*Trimmed reads with at least one reported alignment using RSEM output.

Table 4: Sequencing results of biconcave (Deformed, Def.) and normal (Norm.) vertebra. DE: Differentially Expressed.

although we believe that the study of these non-referenced sequences may improve our understanding of the process involved in deformity appearance.

Gene function analysis using the Ingenuity platform revealed that the top three networks differentially represented in this study were "cellular morphology, embryo development, hair and skin development and function", "organismal development, skeletal and muscular system development and function in cancer" and "hereditary disorder, neurological diseases, organismal injuries and abnormalities". They comprised 30,28 and 28 genes, respectively. In addition, pathway analysis showed that a wide range of canonical pathways were differentially represented $(p<0.05)$. They included, among others, protein kinase A signalling ( $p=1.2 \mathrm{E}-4)$, actin cytoskeleton signalling (6.5E-4), epithelial adherens junction signalling (8.5E-4), axon guidance signalling (1.7E3 ), paxillin signalling (5.4E-3), notch signalling (6.9E-3), inhibition of metalloproteinases (7.8E-3) and FGF signalling (1.9E-2). Enrichment analysis using GOminer highlighted 123 clusters with enrichment $>2$ and $\mathrm{FDR}<5 \%$. Within these clusters are included relevant biological processes, cellular components and molecular functions involved in bone regulation: "GO:0008147 structural constituent of bone": 23.1; "GO:0003308 negative regulation of Wnt receptor signalling pathway involved in heart development": 15.4; "GO:0051019 mitogen-activated protein kinase binding": 13.86; "GO:0030520 estrogen receptor signalling pathway": 6.08; and "GO:0051216 cartilage development": 2.1. Integration of functional analysis coupled to manual screening pointed to putative relevant genes involved in bone metabolism regulation (Table 1).

To understand the mechanisms involved in the depletion of the mineral status linked to the apparition of biconcave vertebrae in P-deficient fish, we combined RNA-seq experiments with recently developed morphometrical techniques. Our first hypothesis was that the lower mineral content and stiffness of deformed vertebrae resulted from higher osteoclast activity (bone resorbing cells). In human osteoporosis, the loss of bone and mineral is commonly linked to higher bone resorption by osteoclasts [49]. Similarly, RANKL-induced ectopic osteoclast activity led to osteoporotic phenotype in medaka (Oryzias latipes), a teleost fish [50]. Mature osteoblasts produce RANKL, which stimulate osteoclast proliferation, differentiation and activation, but they also synthesize osteoprotegerin (OPG), which is the major inhibitor of RANKL/RANK ligation [51]. Here, no differences were observed between both OPG and RANKL gene expression. However, RNA-seq results showed that Tumor Necrosis Factor Receptor Superfamily, Member 5 (TNR5/CD40) was up-regulated in the deformed group.
The ligation to TNR5 receptor induces RANKL pathway activation and bone resorption [52]. To verify whether the lower mineralization in deformed trout vertebrae resulted from higher bone resorption, we verified Tartrate Resistant Acidic Phosphatase (TRAP) gene expression using RT-PCR. Expression of this marker of osteoclast activity was not significantly affected in deformed fish compared to normal individuals (Figure 5). However, in the former, unconventional Myosin 10 was downregulated. Myosin 10 is a crucial protein for podosome belt formation allowing osteoclast attachment [53]. These results are consistent with total bone area assessment using $\mathrm{mCT}$ that did not indicate significant difference between deformed and normal fish (Table 1). Similar bone area profiles are also observed (Figure 3): the higher bone area in the middle area suggesting even lower resorption in deformed vertebrae (Figure 3). Therefore, the lower mineral status in fish with deformed vertebrae did not likely result from higher osteoclast activity. On the contrary, it appeared that low levels of unconventional myosin 10 might impair osteoclast activity after 27 weeks of feeding low-P diet. Thus, our study suggests that impaired vertebral mineralization resulted from either alteration of matrix production and/or mineralization processes.

The mechanisms by which osteoblasts and osteoclasts control bone formation are not fully understood. However, various metabolic pathways affecting bone and cartilage formation have been identified in mammals and recent whole-transcriptome annotation provided evidence that these pathways were also present in teleosts, which suggests high conservation during osteichthyan evolution [26,27]. Genome-wide experiments revealed that most of the pathways (e.g. TGF $\beta / B M P$, WNT and ERK/MAPK pathways) are involved, and may putatively cross-talk, during transcriptional regulation of osteoblast differentiation, proliferation and maturation [54-58]. Our results suggest that the pathways involved in the regulation of osteoblast differentiation and proliferation are down-regulated in deformed fish. Indeed, the following genes were down-regulated in deformed vertebrae: co-receptor activators or receptors (TGF $\beta / B M P$ : BMPR2 WNT: LRP6 and MACF1) and downstream actors (ERK/MAPK: NIK, MEKK1, SOS1, MYCB2 and MYCPP; TGF $/$ BMP: USP9X). Conversely, inhibitors of ligand binding receptor were up-regulated (TGF $\beta / B M P$ : NOG, TEFF1 and FBN2; WNT: SFRP1). For instance, NOG expression was substantially up-regulated (fold change $>9$ ) in deformed compared to normal fish. In transgenic mice, over-expression of NOG triggered osteopenia, i.e. the abnormally low mineral status of bone tissue $[59,60]$. Finally, most of these signal pathway are either directly or indirectly controlled by a master regulator of osteoblast differentiation: RUNX2 [61]. The expression level of RUNX2 was similar in deformed and normal vertebrae, but conflicting results were obtained regarding RUNX2 regulation. Indeed, in deformed fish we observed a lower expression level of HIVEP3, a gene negatively regulating osteoblast differentiation by stimulating degradation of RUNX2 and acting as a damper for signalling pathways such as WNT, TGF $\beta$ and BMP2 [62-66]. TWIST2, a RUNX2 inhibitor, was up-regulated in deformed fish while activators, such as $S T A B 2$, were down-regulated [67]. In vitro experiments have shown that osteoblastogenesis is a physiological process that spends several weeks, during which specific actors are expressed at different times/stages [68]. Yet, in deformed fish RUNX2 expression was not significantly affected, a finding that may be consistent with the higher expression of osteocalcin (also known as bone Gla protein, BGLAP), which normally occurs several weeks after a peak of RUNX2 [69].

$B G L A P$ expression is a marker of mature osteoblasts. In deformed fish, the higher expression of BGLAP is concomitant with the higher 

Vertebrae: A Common Abnormality in Rainbow Trout (Oncorhynchus mykiss, Walbaum) Fed a Low-Phosphorus Diet. Next Generat Sequenc \& Applic 2: 112. doi:10.4172/2469-9853.1000112

expression of JUND, an AP-1 transcription factor complex, responsible for binding to BGLAP promoter and enhancing BGLAP expression in mammalian osteoblasts [70]. In vitro experiments showed that JUND expression was relatively low during osteoblast proliferation and then increased during matrix maturation to reach a peak during matrix mineralization [71].

Taken together our analyses of biconcave vertebrae of deformed fish suggest that the processes involved in osteoblast differentiation and proliferation are reduced while the high level of BGLAP expression indicates the presence of numerous mature osteoblasts. We observed consistently in these deformed fish a low expression of COL12a1, a collagen type highly expressed by differentiating osteoblasts but not in mature osteoblasts, in which its expression is reduced [72]. Furthermore, previous studies report that COL12a1 knockout mice displayed altered vertebral structure with decreased bone strength [73], which is coherent with the lower bone stiffness observed in deformed fish. Interestingly, the same authors reported no difference in TRAP expression or in other markers of bone resorption in COL12a1 -/- mice vs. wild-type control, indicating that decreased bone mass resulted from abnormal bone formation rather than from changes in bone resorption [73]. The expression of COL11a1, a gene coding for a structural component of cartilage matrix, trabecular and compact bone as well as notochord tissue was also down-regulated in deformed fish compared to normal individuals. In mammals, COL11a1 ensures cohesiveness of collagen matrix and its altered expression results in thicker fibers and increased mineralization [74]. In fish, COL11a1 is mostly present in notochord tissue, and is also expressed by osteoblasts in trabecular bone [25] Interestingly, in Atlantic salmon a long photoperiod resulted in a lower amount of COL11al in compact bone of compressed vertebrae [25]. COL11a1 expression was also reduced in Atlantic salmon subjected to a combination of low P-dietary treatment and long photoperiod [75].

Nevertheless, at the histological level our study demonstrated that both trabecular bone area and the surface of mineralized bone areas were not significantly different ( $p=0.39$ and 0.32 , respectively) in pronounced biconcave compared to normal vertebrae (Table 3). We conclude that if the capability of osteoblasts to form new osteoid was not differentially affected in P-deficient fish with either biconcave or normal vertebrae, then the observed differences in mineral content might thus rather be related to the alteration of mineralization degree of the bone matrix.

Bone mineralization corresponds to the precipitation of calcium phosphate as hydroxyapatite (HA) crystals within a collagen-rich matrix, although how mineral formation is controlled is not fully understood. The combination of a favourable environment along with removal of mineralization inhibitors are known to be key factors regulating crystal formation [76]. Similarly, the interaction of HA crystals with the organic matrix and their growth and expansion have not been fully elucidated.

However, we know that bone matrix, including non-collagenous proteins such as BGLAP, matrix Gla protein (MGP), osteopontin (also known as secreted phosphoprotein 1, SPP1) and SPARC, also control mineral deposition in bone tissue [77,78]. As said above BGLAP expression was higher in biconcave than in normal vertebra with regard to RNA-seq experiment as well as by confirmation with RT-qPCR (Figure 5). In mammals BGLAP is known to be expressed exclusively by mature osteoblasts and osteocytes $[68,70]$ and constitutes the major non-collagenous protein in bone tissue. The role of circulating BGLAP is particularly relevant for energy metabolism and glycemia regulation [79]. Interestingly, we observed a substantial up-regulation (FC $>34)$ of GCG2, involved in lipid and glycogen hydrolysis and higher calcium intake [80]. However, despite extensive studies, the direct role of BGLAP in bone tissue is still subject to debate. For instance, BGLAPdeficient mice displayed no detectable impact on bone mineralization using Von Kossa staining [81], but infrared microspectrometry showed its positive role in the maturation of hydroxyapatite crystals [82]. In juvenile Atlantic salmon with high growth rate BGLAP expression was negatively affected and the activity of mature osteoblasts and mineralization were reduced [24]. Low BGLAP expression could result in a default in mature osteoblast function, hence triggering default in mineralization status. However, by controlling the direction of crystal growth or even inhibiting it, high BGLAP expression may also have negative impacts on mineralization $[77,83,84]$. The up-regulation of $B G L A P$ is also consistent with a higher expression of C/EBP- $\beta$, a putative positive regulator of $B G L A P$, a finding which was also reported in rat [85]. Interestingly, vitamin $\mathrm{D}$ is known to be a regulator of $\mathrm{C} /$ EBP- $\beta$ expression and has also been involved in regulation of BGLAP expression $[70,86]$. Similarly, vitamin D and expression of its receptor, $V D R$ are affected by P-level in the diet $[48,75,87,88]$. In rainbow trout vitamin D induces $\mathrm{NaPi}-\mathrm{II}$ expression, which is responsible for $\mathrm{P}$ absorption and retention [89]. However, conflicting responses of $\mathrm{P}$ dynamics to vitamin $\mathrm{D}$ treatments have been observed in fish, indicating differences between teleosts and mammals [87,89]. MGP, encoding a protein involved in the regulation of the mineralization process, was up-regulated in deformed fish. MGP is a potent inhibitor of bone and cartilage mineralization [90,91]. In contrast to BGLAP, MGP is expressed in various tissues and is not limited to bone tissue in Atlantic salmon [92]. The expression of MGP in deformed fish is negatively correlated to bone ash content and mechanical quality (stiffness) in deformed fish.

The ossification by matrix proteins was related to expression of DCHS1 and FAT4, both being lower in deformed than in normal fish. Interestingly, studies in transgenic mice indicated that the two proteins act as ligand-receptor complexes; Mice in which DCHS1 and FAT4 were invalidated show defects in vertebral column development and abnormal patterns of skeletal ossification [93]. The mechanisms by which these proteins play a role in skeletal tissue are not fully understood. However, according to the authors, DCHS1 and FAT4 could play a role in interacting with planar cell polarity effector, a pathway that was significantly impacted in the current study. Taken together these results highlight various actors, including matrix proteins that could be involved in the default of degree of mineralization observed in deformed fish and thus putatively triggering the development of deformities.

Finally, under normal conditions, the teleost skeleton displays a wide spectrum of intermediate skeletal tissues (from cartilage-like to bone-like tissues), which are complex to study [94,95]. In teleost vertebrae, more or less mineralized healthy cartilage as well as ectopic cartilage can be observed, the former being related to a metaplastic shift of bone and co-transcription of chondrocytic and osteogenic markers $[23,24,96,97]$. During development, the cartilage-type tissue localized in the basalia is replaced by bone-type tissue by a process called endochondral ossification. This process requires chondrocyte hypertrophy and apoptosis, blood vessel invasion and ECM degradation. It was thus important to assess whether molecular activity or overall mineral status might be biased by the high cellular activity within the basalias. Both osteoblasts and chondrocytes differentiate from mesenchymal stem cells and thus share numerous key events 

Vertebrae: A Common Abnormality in Rainbow Trout (Oncorhynchus mykiss, Walbaum) Fed a Low-Phosphorus Diet. Next Generat Sequenc \& Applic 2: 112. doi:10.4172/2469-9853.1000112

Page 10 of 13

involved in their differentiation and regulation [26]. Here, we observed that pathways involved in chondrocyte differentiation were affected in deformed fish (down-regulation of SHH and FGFR4) but not the expression level of a key mediator of cartilage formation SOX9 [98] and of $M E F 2 C$, a marker of chondrogenesis [23,99], which remained constant. Expression of $M E F 2 C$ was further confirmed by RT-qPCR (Figure 5). Furthermore, $N K X 3.2$, a transducer of $\mathrm{SOX} 9$ signals was upregulated in deformed fish $[67,100]$. Our results also suggest that the invasion of blood vessels in the cartilage matrix, its degradation and replacement by osteoblasts might be negatively impacted in deformed fish, even if a distinctive phenotype is lacking. Indeed, deformed fish displayed impairment of the VEGF signalling pathway as indicated by lower expression of HSPG2, GPC1 and VEGFR1. The VEGF pathway is involved in angiogenesis (invasion of blood vessels) and ossification [101-103]. HSPG2 -/- mice displayed strong impairment of cartilage formation, ultimately leading to diminished osteoblast replacement and endochondral bone formation [104]. Interestingly, in deformed fish we did not observe differences in the mineralization status of basalia. Consistent with observations of HSPG2 -/- mice, MMP10 expression was increased in deformed fish. MMP10 is expressed in chondrocytes degrading the cartilage matrix as well as in some, but not all osteoclasts and is also responsible for activation of several other MMPs including MMP9, that is involved in the vascularization process of the growth plate [105-107]. Here however, we observed an over-representation of canonical pathways for the inhibition of MMPs, hence the complexity to interpret the role of MMPs in deformed vertebrae. Thus, our results suggest that basalia deformed vertebrae might experience a subsequent endochondral ossification but with no impact of the calcification of the basalia by the time of sampling. To confirm the gene expression results, we used Sirius red staining coupled with image processing to extract the basalias and quantitatively assess the presence of mature collagen fibers (putatively mineralized) and that of less mature collagen fibers. In both groups, we observed that the vertebral arches were not fully ossified, but no significant differences were observed in their mineralization status (Table 3). Furthermore, no obvious presence of ectopic cartilage or remodelling of the notochord was observed in either deformed or normal fish, as previously reported in severe compression type deformities in other salmonids $[24,96,108]$.

\section{Conclusion}

Interpreting data from RNA-seq experiments, particularly in a complex tissue like vertebra, remains challenging and is limited to the gene expression levels. This approach however, provides an unprecedented overview regarding molecular changes that are involved in the occurrence of vertebral deformities in P-deficient trout. The aim of our study was not to describe the impact of P-deficiency in trout but rather to characterize discrete differences between fish fed low-P diets presenting either deformed or normal vertebral phenotypes. We observed that the initial appearance of malformations in rainbow trout is associated with changes in numerous signalling pathways similar to those described in mammals, confirming their evolutionary conservation. Our results also suggested that after 27 weeks feeding low $\mathrm{P}$ diets, deformed vertebrae showed a decrease in osteoblast differentiation and proliferation while they display a higher expression of BGLAP, a marker of mature osteoblast activity. Together with histological observations, this suggests that during the weeks prior to sampling, deformed fish were still differentiating osteoblasts and producing matrix. However, higher expression of MGP and BGLAP as well as lower circulating $\mathrm{P}$ significantly impaired mineralization. Furthermore, impaired cohesiveness together with low mineralization reduced vertebrae stiffness. Consequently, reduced vertebral integrity might increase vertebrae susceptibility to deform under normal physical forces. The conserved osteoclast activity together with the production of BGLAP and GCG2 suggests that deformed vertebrae are undergoing significant bone remodelling with high bone resorption process not yet observable in our study. This process may lead to new vertebral phenotypes. As suggested in previous studies [20,28], pronounced biconcave vertebrae may be a transitory step toward a more dramatic abnormal vertebral phenotype (homogeneous compressed). Similar analysis of vertebral gene expression over time will highlight molecules involved in the occurrence of vertebral deformities. Our study provides consistent evidence of the importance of the matrix proteins and other markers involved in reduced mineralization and mechanical strength of vertebra, recognised as initial indicators of deformity development when feeding low-P diets to fish [48]. Previous family-based studies in fish showed that genetics has an impact on the prevalence of deformities [109-111]. With the aim of reducing $P$ excretion to promote sustainable freshwater aquaculture production, the indicators revealed in our study may ultimately serve as markers for genetic selection of fish strains displaying normal growth performance with a low prevalence of deformities, when feeding low-P diets.

\section{Acknowledgement}

We greatly thank the technical assistance of the Laboratoire des sciences aquatiques de l'Université Laval and Dr. Brian Boyle for his assistance with the Illumina library preparation at the Institut de Biologie Intégrative des Systèmes (IBIS) Québec. We thank as well Éric Normandeau for his help in bioinformatics analyses. Computations were carried out on the supercomputer Colosse, Université Laval managed by Calcul Québec and Compute Canada. The assistance of Benjamin Bourdon regarding biomechanical analyses of vertebrae is acknowledged. This project was supported by the Ministère du Développement économique, Innovation et Exportation du Québec, the Ressources Aquatiques Québec (RAQ), the Sociéte de recherche et de développement en aquaculture continentale (SORDAC) Inc. (PSR-SIIRI-443) and the Aquaculture Collaborative Research and Development Program, Fisheries and Oceans Canada (Q-08-01-001).

\section{References}

1. Boglione C, Gavaia P, Koumoundouros G, Gisbert E, Moren M, et al. (2013) Skeletal anomalies in reared European fish larvae and juveniles. Part 1: normal and anomalous skeletogenic processes. Rev Aquac 5: S99-S120.

2. Boglione C, Gavaia P, Koumoundouros G, Gisbert E, Moren MM, et al. (2013) Skeletal anomalies in reared European fish larvae and juveniles. Part 2: main typologies, occurrences and causative factors. Rev Aquac 5: S121-S167.

3. Fjelldal PG, Hansen T, Breck O, Ørnsrud R, Lock E-J, et al. (2012) Vertebra deformities in farmed Atlantic salmon (Salmo salar L.) - etiology and pathology. J Appl Ichthyol 28: 433-440.

4. Sugiura SH, Hardy RW, Roberts RJ (2004) The pathology of phosphorus deficiency in fish--a review. J Fish Dis 27: 255-265.

5. Lall SP (2002) The minerals. In Fish Nutrition. Third. Edited by Halver JE, Hardy RW. San diego: Elsevier Academic Press: 259-308.

6. Lall SP, Lewis-McCrea LM (2007) Role of nutrients in skeletal metabolism and pathology in fish--an overview. Aquaculture 267: 3-19.

7. Hua K (2005) Modeling phosphorus utilization in salmonid fish species University of Guelph; pp212.

8. Rønsholdt B (1995) Effect of size/age and feed composition on body composition and phosphorus content of rainbow trout, Oncorhynchus mykiss. Water Sci Technol 31: 175-183.

9. Francillon-Vieillot $\mathrm{H}$, de Buffrénil V, Castanet J, Géraudie J, Meunier FJJ, et al. (1990) Microstructure and mineralization of vertebrate skeletal tissues. In Skeletal Biomineralization: Patterns, Processes and Evolutionary trends. Carter, J. Edited by Carter JG. Van Nostrand Reinhold, New York: Van Nostran Reinhold, New York, USA: 471-530

10. Vielma J (1998) Phosphorus utilization by Atlantic salmon (Salmo salar) reared in freshwater is not influenced by higher dietary calcium intake. Aquaculture 160: 117-128. 
Citation: Le Luyer J, Deschamps MH, Proulx E, Poirier Stewart N, Droit A, et al. (2015) RNA-Seq Transcriptome Analysis of Pronounced Biconcave Vertebrae: A Common Abnormality in Rainbow Trout (Oncorhynchus mykiss, Walbaum) Fed a Low-Phosphorus Diet. Next Generat Sequenc \& Applic 2: 112. doi:10.4172/2469-9853.1000112

11. Kacem A, Gustafsson S, Meunier FJ (2000) Demineralization of the vertebral skeleton in Atlantic salmon Salmo salar L. during spawning migration. A Mo Integr Physiol 125: 479-484.

12. Kacem A, Meunier FJ (2003) Halastatic demineralization in the vertebrae of Atlantic salmon, during their spawning migration. J Fish Biol 63: 1122-1130.

13. Skonberg DI, Yogev L, Hardy RW, Dong FM (1997) Metabolic response to dietary phosphorus intake in rainbow trout (Oncorhynchus mykiss). Aquaculture 157: $11-24$

14. Baeverfjord G, Åsgård T, Shearer KD (1998) Development and detection of phosphorus deficiency in Atlantic salmon,Salmo salar L., parr and post-smolts Aquac Nutr 4: 1-11.

15. Deschamps MH, Poirier Stewart N, Demanche A, Vandenberg GW. (2014) Preliminary study for description of bone tissue responsiveness to prolonged dietary phosphorus deficiency in rainbow trout, Oncorhynchus mykiss (Walbaum). Aquac Res: 1-12

16. Lellis WA, Barrows FT, Hardy RW (2004) Effects of phase-feeding dietary phosphorus on survival, growth, and processing characteristics of rainbow trout Oncorhynchus mykiss. Aquaculture 242: 607-616.

17. Helland S, Refstie S, Espmark A, Hjelde K, Baeverfjord Get al. (2005) Minera balance and bone formation in fast-growing Atlantic salmon parr (Salmo salar) in response to dissolved metabolic carbon dioxide and restricted dietary phosphorus supply. Aquaculture 250: 364-376

18. Deschamps MH, Poirier Stewart N, Demanche A, Vandenberg GW (2014) Preliminary study for phenotypic description of vertebral abnormalities in trout subjected to prolonged deficiency in phosphorus. J Appl Ichthyol 30: 833-839.

19. Poirier Stewart N, Deschamps MH, Witten PE, Le Luyer J, Proulx E, et al. (2014) X-ray-based morphometrics: an approach to diagnose vertebral abnormalities in under-mineralized vertebrae of juvenile triploid all-female rainbow trout (Oncorhynchus mykiss) fed with a phosphorus deficient diet. $J$ Appl Ichthyol 30:796-803

20. Franz-Odendaal TA, Hall BK, Witten PE (2006) Buried alive: how osteoblasts become osteocytes. See comment in PubMed Commons below Dev Dyn 235 176-190.

21. Witten PE, Huysseune A (2009) A comparative view on mechanisms and functions of skeletal remodelling in teleost fish, with special emphasis on osteoclasts and their function. Biol Rev Camb Philos Soc 84: 315-346.

22. Ytteborg E, Baeverfjord G, Torgersen J, Hjelde K, Takle H (2010) Molecular pathology of vertebral deformities in hyperthermic Atlantic salmon (Salmo salar). BMC Physiol 10: 12

23. Ytteborg E, Torgersen J, Baeverfjord G, Takle H (2010) Morphological and molecular characterization of developing vertebral fusions using a teleost model.BMC Physiol 10: 13

24. Wargelius A, Fjelldal PG, Nordgarden U, Grini A, Krossøy C, et al. (2010) Collagen type $\mathrm{XI}$ alpha1 may be involved in the structural plasticity of the vertebral column in Atlantic salmon (Salmo salar L.). J Exp Biol 213: 12071216.

25. Vieira FA, Thorne MA, Stueber K, Darias M, Reinhardt R, et al. (2013) Comparative analysis of a teleost skeleton transcriptome provides insight into its regulation.Gen Comp Endocrinol 191: 45-58.

26. Le Luyer J, Deschamps MH, Proulx E, Poirier Stewart N, Joly Beauparlant C (2014) Establishment of a comprehensive reference transcriptome for vertebra bone tissue to study the impacts of nutritional phosphorus deficiency in rainbow trout (Oncorhynchus mykiss, Walbaum) Mar Genomics 18: 141-144.

27. Witten PE, Gil-Martens L, Huysseune A, Takle H, Hjelde K (2009) Towards a classification and an understanding of developmental relationships of vertebra body malformations in Atlantic salmon (Salmo salar L.). Aquaculture 295: 6-14.

28. Coleman R (2011) Picrosirius red staining revisited. Acta Histochem 113 231-233

29. Rungby J, Kassem M, Eriksen EF, Danscher G (1993) The von Kossa reaction for calcium deposits: silver lactate staining increases sensitivity and reduces background. Histochem J 25: 446-451.

30. Ruifrok AC, Johnston DA (2001) Quantification of histochemical staining by color deconvolution. Anal Quant Cytol Histol 23: 291-299.

31. Deschamps MH, Girondot M, Labbé L, Sire JY (2009) Changes in vertebral structure during growth of reared rainbow trout, Oncorhynchus mykiss
(Walbaum): a new approach using modelling of vertebral bone profiles. J Fish Dis 32: $233-246$

32. Girondot M, Laurin M (2003) Bone Profiler: a tool to quantify, model and statistically compare bone section compactness profile. J Vertebr Paleontol 23 : 458-461.

33. Fjelldal PG, Grotmol S, Kryvi H, Gjerdet NR, Taranger GL, et al. (2004) Pinealectomy induces malformation of the spine and reduces the mechanical strength of the vertebrae in Atlantic salmon (Salmo salar). J Pineal Res 36 132-139.

34. Lohse M, Bolger AM, Nagel A, Fernie AR, Lunn JE, et al. (2012) RobiNA: a user-friendly, integrated software solution for RNA-Seq-based transcriptomics. Nucleic Acids Res 40: W622-627.

35. Haas BJ, Papanicolaou A, Yassour M, Grabherr M, Blood PD, et al. (2013) De novo transcript sequence reconstruction from RNA-seq using the Trinity platform for reference generation and analysis. Nat Protoc 8: 1494-1512.

36. Li B, Dewey CN (2011) RSEM: accurate transcript quantification from RNA-Seq data with or without a reference genome.BMC Bioinformatics 12: 323 .

37. Langmead B, Trapnell C, Pop M, Salzberg SL (2009) Ultrafast and memoryefficient alignment of short DNA sequences to the human genome. Genome Biol 10: R25.

38. Robinson MD, McCarthy DJ, Smyth GK (2010) edgeR: a Bioconducto package for differential expression analysis of digital gene expression data. Bioinformatics 26: 139-140.

39. Anders S, Huber W (2010) Differential expression analysis for sequence count data. Genome Biol 11: R106.

40. Zeeberg BR, Feng W, Wang G, Wang MD, Fojo AT, et al. (2003) GoMiner: a resource for biological interpretation of genomic and proteomic data. Genome Biol 4:R28.

41. Li A, Zhang J, Zhou Z (2014) PLEK: a tool for predicting long non-coding RNAs and messenger RNAs based on an improved k-mer scheme. BMC Bioinformatics 15: 311

42. Morrison TB, Weis JJ, Wittwer CT (1998) Quantification of low-copy transcripts by continuous SYBR Green I monitoring during amplification.Biotechniques 24 954-958, 960, 962

43. Vandesompele J, De Preter K, Pattyn F, Poppe B, Van Roy N, et al. (2002) Accurate normalization of real-time quantitative RT-PCR data by geometric averaging of multiple internal control genes. Genome Biol 3: RESEARCH0034.

44. Dean CB, Nielsen JD (2007) Generalized linear mixed models: a review and some extensions.Lifetime Data Anal 13: 497-512.

45. Komsta L, Novomestky F (2012) moments: Moments, cumulants, skewness, kurtosis and related tests. Available at http://CRAN.R-project.org/ package $=$ moments

46. Fjelldal PG, Lock EJ, Grotmol S, Totland GK, Nordgarden U, et al. (2006) Impact of smolt production strategy on vertebral growth and mineralisation during smoltification and the early seawater phase in Atlantic salmon (Salmo salar, L.). Aquaculture 261: 715-728

47. Fjelldal PG, Hansen T, Breck O, Sandvik R, Waagbø R, et al. (2009) Supplementation of dietary minerals during the early seawater phase increase vertebral strength and reduce the prevalence of deformities in fast-growing under-yearling Atlantic salmon (Salmo salar, L.) smolt. Aquac Nutr 15: 366-378.

48. Mizuno A, Kanno T, Hoshi M, Shibata O, Yano K, et al. (2002) Transgenic mice overexpressing soluble osteoclast differentiation factor (sODF) exhibit severe osteoporosis. J Bone Miner Metab 20: 337-344.

49. To TT, Witten PE, Renn J, Bhattacharya D, Huysseune A, et al. (2012) Ranktinduced osteoclastogenesis leads to loss of mineralization in a medaka osteoporosis model. See comment in PubMed Commons below Development 139: $141-150$.

50. Boyce BF, Xing L (2008) Functions of RANKL/RANK/OPG in bone modeling and remodeling. Arch Biochem Biophys 473: 139-146.

51. Lee HY, Jeon HS, Song EK, Han MK, Park SI, et al. (2006) CD40 ligation of rheumatoid synovial fibroblasts regulates RANKL-mediated osteoclastogenesis: evidence of NF-kappaB-dependent, CD40-mediated bone destruction in rheumatoid arthritis. Arthritis Rheum 54: 1747-1758.

52. McMichael BK, Cheney RE, Lee BS (2010) Myosin X regulates sealing zone 
Citation: Le Luyer J, Deschamps MH, Proulx E, Poirier Stewart N, Droit A, et al. (2015) RNA-Seq Transcriptome Analysis of Pronounced Biconcave Vertebrae: A Common Abnormality in Rainbow Trout (Oncorhynchus mykiss, Walbaum) Fed a Low-Phosphorus Diet. Next Generat Sequenc \& Applic 2: 112. doi:10.4172/2469-9853.1000112

patterning in osteoclasts through linkage of podosomes and microtubules. $J$ Biol Chem 285: 9506-9515.

53. Aubin J, Davy A, Soriano P (2004) In vivo convergence of BMP and MAPK signaling pathways: impact of differential Smad1 phosphorylation on development and homeostasis. Genes Dev 18: 1482-1494.

54. Zamurovic N, Cappellen D, Rohner D, Susa M (2004) Coordinated activation of notch, Wnt, and transforming growth factor-beta signaling pathways in bone morphogenic protein 2-induced osteogenesis. Notch target gene Hey1 inhibits mineralization and Runx2 transcriptional activity. J Biol Chem 279: 37704 37715

55. Ornitz DM, Marie PJ (2002) FGF signaling pathways in endochondral and intramembranous bone development and human genetic disease.Genes Dev 16: $1446-1465$

56. Ge C, Xiao G, Jiang D, Franceschi RT (2007) Critical role of the extracellular signal-regulated kinase-MAPK pathway in osteoblast differentiation and skeletal development. J Cell Biol 176: 709-718.

57. Glass DA , Karsenty G (2007) In vivo analysis of Wnt signaling in bone Endocrinology 148: 2630-2634

58. Devlin RD, Du Z, Pereira RC, Kimble RB, Economides AN, et al. (2003) Skeletal overexpression of noggin results in osteopenia and reduced bone formation. Endocrinology 144: 1972-1978.

59. Wu X, Li Y, Schneider A, Yu W, Rajendren G, et al. (2003) Impaired osteoblastic differentiation, reduced bone formation, and severe osteoporosis in nogginoverexpressing mice. J Clin Investig 112: 924-934.

60. Franceschi RT, Xiao G (2003) Regulation of the osteoblast-specific transcription factor, Runx2: responsiveness to multiple signal transduction pathways. J Cell Biochem 88: 446-454.

61. Bodine PV, Komm BS (2006) Wnt signaling and osteoblastogenesis.Rev Endocr Metab Disord 7: 33-39.

62. Piters E, Boudin E, Van Hul W (2008) Wnt signaling: a win for bone. Arch Biochem Biophys 473: 112-116.

63. Bonewald LF, Johnson ML (2008) Osteocytes, mechanosensing and Wnt signaling.Bone 42: 606-615

64. Imamura K, Maeda S, Kawamura I, Matsuyama K, Shinohara N, et al. (2014) Human immunodeficiency virus type 1 enhancer-binding protein 3 is essential for the expression of asparagine-linked glycosylation 2 in the regulation of osteoblast and chondrocyte differentiation. J Biol Chem 289: 9865-9879.

65. Shim JH, Greenblatt MB, Zou W, Huang Z, Wein MN, et al. (2013) Schnurri-3 regulates ERK downstream of WNT signaling in osteoblasts. J Clin Invest 123 4010-4022.

66. Karsenty G (2008) Transcriptional control of skeletogenesis. Annu Rev Genomics Hum Genet 9: 183-196.

67. Beck GR Jr, Moran E, Knecht N (2003) Inorganic phosphate regulates multiple genes during osteoblast differentiation, including Nrf2. Exp Cell Res 288: 288300 .

68. Karsenty G (2000) Bone formation and factors affecting this process. Matrix Biol 19: 85-89.

69. Yeung F, Law WK, Yeh CH, Westendorf JJ, Zhang Y, et al. (2002) Regulation of human osteocalcin promoter in hormone-independent human prostate cancer cells. J Biol Chem 277: 2468-2476.

70. McCabe LR, Kockx M, Lian J, Stein J, Stein G (1995) Selective expression of fos- and jun-related genes during osteoblast proliferation and differentiation. Exp Cell Res 218: 255-262.

71. Granchi D, Ochoa G, Leonardi E, Devescovi V, Baglio SR, et al. (2010) Gene expression patterns related to osteogenic differentiation of bone marrowderived mesenchymal stem cells during ex vivo expansion. Tissue Eng Part $\mathrm{C}$ Methods 16: $511-524$

72. Izu Y, Sun M, Zwolanek D, Veit G, Williams V, et al. (2011) Type XII collagen regulates osteoblast polarity and communication during bone formation. J Cell Biol 193: 1115-1130.

73. Li Y, Lacerda DA, Warman ML, Beier DR, Yoshioka H, et al. (1995) A fibrilla collagen gene, Col11a1, is essential for skeletal morphogenesis. Cell 80: 423430
74. Fjelldal PG, Lock EJ, Hansen T, Waagbø R, Wargelius A, et al. (2012) Continuous light induces bone resorption and affects vertebral morphology in Atlantic salmon (Salmo salar L.) fed a phosphorous deficient diet. Aquac Nutr 18: $610-619$.

75. Pasteris JD, Wopenka B, Valsami Jones E (2008) Bone and Tooth Mineralization: Why Apatite? Elements 4: 97-104

76. Boskey AL, Robey PG (2013) The Regulatory Role of Matrix Proteins in Mineralization of Bone. Fourth Edi. Elsevier: 235-255.

77. Hall BK (2005) Osteoblast and osteocyte diversity. In Bones and Cartilage. Edited by Hall BK. San Diego: Elsevier Academic Press; 329-337.

78. Karsenty G, Ferron M (2012) The contribution of bone to whole-organism physiology. Nature 481: 314-320.

79. Haderslev KV, Jeppesen PB, Hartmann B, Thulesen J, Sorensen HA, et al. (2002) Short-term administration of glucagon-like peptide-2. Effects on bone mineral density and markers of bone turnover in short-bowel patients with no colon. Scand J Gastroenterol 37: 392-398.

80. Ducy P, Desbois C, Boyce B, Pinero G, Story B, et al. (1996) Increased bone formation in osteocalcin-deficient mice. Nature 382: 448-452.

81. Boskey AL, Gadaleta S, Gundberg C, Doty SB, Ducy P, et al. (1998) Fourier transform infrared microspectroscopic analysis of bones of osteocalcin-deficient mice provides insight into the function of osteocalcin. Bone 23: 187-196.

82. Hunter GK, Hauschka PV, Poole AR, Rosenberg LC, Goldberg HA (1996) Nucleation and inhibition of hydroxyapatite formation by mineralized tissue proteins. Biochem J 317: 59-64.

83. Roy ME, Nishimoto SK, Rho JY, Bhattacharya SK, Lin JS, et al. (2001) Correlations between osteocalcin content, degree of mineralization, and mechanical properties of C. carpio rib bone. J Biomed Mater Res 54: 547-553.

84. Gutierrez S, Javed A, Tennant DK, van Rees M, Montecino M, et al. (2002) CCAAT/enhancer-binding proteins (C/EBP) beta and delta activate osteocalcin gene transcription and synergize with Runx2 at the C/EBP element to regulate bone-specific expression. J Biol Chem 277: 1316-1323.

85. Lian JB, Stein GS, Stein JL, van Wijnen AJ (1998) Osteocalcin gene promoter unlocking the secrets for regulation of osteoblast growth and differentiation. J Cell Biochem Suppl 30-31: 62-72.

86. Lock EJ, Waagb ø R, Wendelaar Bonga S, Flik G (2010) The significance of vitamin $D$ for fish: a review. Aquac Nutr 16: 100-116.

87. Meyer J, Fullmer CS, Wasserman RH, Komm BS, Haussler MR (1992) Dietary restriction of calcium, phosphorus, and vitamin $D$ elicits differential regulation of the mRNAs for avian Intestinal calbindin-D28k and the 1,25-dihydroxyvitamin D, receptor. J Bone Miner Res 7: 441-448.

88. Sugiura SH, Ferraris RP (2004) Dietary phosphorus-responsive genes in the intestine, pyloric ceca, and kidney of rainbow trout. Am J Physiol Regul Integr Comp Physiol 287: R541-550.

89. Julien M, Khoshniat S, Lacreusette A, Gatius M, Bozec A, et al (2009) Phosphate-dependent regulation of MGP in osteoblasts: role of ERK1/2 and Fra-1. J Bone Miner Res 24: 1856-1868.

90. Gopalakrishnan R, Ouyang H, Somerman MJ, McCauley LK, Francesch RT (2001) Matrix gamma-carboxyglutamic acid protein is a key regulator of PTH-mediated inhibition of mineralization in MC3T3-E1 osteoblast-like cells Endocrinology 142: 4379-4388.

91. Krossøy C, Ornsrud R, Wargelius A (2009) Differential gene expression of bgp and mgp in trabecular and compact bone of Atlantic salmon (Salmo salar L.) vertebrae. J Anat 215: 663-672.

92. Mao Y, Mulvaney J, Zakaria S, Yu T, Morgan KM, et al. (2011) Characterization of a Dchs1 mutant mouse reveals requirements for Dchs1-Fat4 signaling during mammalian development. Development 138: 947-957.

93. Witten PE, Huysseune A, Hall BK (2010) A practical approach for the identification of the many cartilaginous tissues in teleost fish. J Appl Ichthyol 26: $257-262$

94. Apschner A, Schulte-Merker S, Witten PE (2011) Not All Bones are Created Equal - Using Zebrafish and Other Teleost Species in Osteogenesis Research In Methods in cell biology. Volume 105. Third Edit. Elsevier Inc: 239-255.

95. Witten PE, Gil-Martens L, Hall BK, Huysseune A, Obach A (2005) Compressed vertebrae in Atlantic salmon Salmo salar: evidence for metaplastic 
Citation: Le Luyer J, Deschamps MH, Proulx E, Poirier Stewart N, Droit A, et al. (2015) RNA-Seq Transcriptome Analysis of Pronounced Biconcave Vertebrae: A Common Abnormality in Rainbow Trout (Oncorhynchus mykiss, Walbaum) Fed a Low-Phosphorus Diet. Next Generat Sequenc \& Applic 2: 112. doi:10.4172/2469-9853.1000112

Page 13 of 13

chondrogenesis as a skeletogenic response late in ontogeny. Dis Aquat Organ 64: $237-246$

96. Ytteborg E, Torgersen J, Baeverfjord G, Takle H (2012) Four stages characterizing vertebral fusions in Atlantic salmon. J Appl Ichthyol 28: 453-459.

97. de Crombrugghe B, Lefebvre V, Nakashima K (2001) Regulatory mechanisms in the pathways of cartilage and bone formation. Curr Opin Cell Biol 13: 721-727.

98. Arnold MA, Kim Y, Czubryt MP, Phan D, McAnally J, et al. (2007) MEF2C transcription factor controls chondrocyte hypertrophy and bone development. Dev Cell 12: 377-389.

99. Zeng L, Kempf H, Murtaugh LC, Sato ME, Lassar AB (2002) Shh establishes an $\mathrm{Nkx} 3.2 /$ Sox 9 autoregulatory loop that is maintained by BMP signals to induce somitic chondrogenesis. Dev 16: 1990-2005.

100. Ferrara N, Gerber HP, LeCouter J (2003) The biology of VEGF and its receptors. Nat Med 9: 669-676.

101. Gerber HP, Vu TH, Ryan AM, Kowalski J, Werb Z, et al. (1999) VEGF couples hypertrophic cartilage remodeling, ossification and angiogenesis during endochondral bone formation. Nat Med 5: 623-628.

102. Murata M, Yudoh K, Masuko K (2008) The potential role of vascular endothelial growth factor (VEGF) in cartilage: how the angiogenic factor could be involved in the pathogenesis of osteoarthritis? Osteoarthritis Cartilage 16: 279-286.

103. Ishijima M, Suzuki N, Hozumi K, Matsunobu T, Kosaki K, et al. (2012) Perlecan modulates VEGF signaling and is essential for vascularization in endochondral bone formation. Matrix Biol 31: 234-245.
104. Batra J, Robinson J, Soares AS, Fields AP, Radisky DC, et al. (2012) Matrix metalloproteinase-10 (MMP-10) interaction with tissue inhibitors of metalloproteinases TIMP-1 and TIMP-2: binding studies and crystal structure. J Biol Chem 287: 15935-15946.

105. Kevorkian L, Young DA, Darrah C, Donell ST, Shepstone L, et al. (2004) Expression profiling of metalloproteinases and their inhibitors in cartilage. Arthritis Rheum 50: 131-141.

106. Engsig MT, Chen QJ, Vu TH, Pedersen AC, Therkidsen B, et al. (2000) Matrix metalloproteinase 9 and vascular endothelial growth factor are essential for osteoclast recruitment into developing long bones. J Cell Biol 151: 879-889.

107. Witten PE, Obach A, Huysseune A, Baeverfjord G (2006) Vertebrae fusion in Atlantic salmon (Salmo salar): development, aggravation and pathways of containment. Aquaculture 258: 164-172.

108. Gislason H, Karstensen H, Christiansen D, Hjelde K, Helland S, et al. (2010) $\mathrm{Rib}$ and vertebral deformities in rainbow trout (Oncorhynchus mykiss) explained by a dominant-mutation mechanism. Aquaculture 309: 86-95.

109. McKay LR, Gjerde B (1986) Genetic variation for a spinal deformities in Atlantic salmon, Salmo salar. Aquaculture 52: 263-272.

110. Gjerde B, Pante M, Josefa R, Baeverfjord G (2005) Genetic variation for a vertebral deformity in Atlantic salmon (Salmo salar). Aquaculture 244: 77-87.

111. Kolstad K, Thorland I, Refstie T, Gjerde B (2006) Genetic variation and genotype by location interaction in body weight, spinal deformity and sexual maturity in Atlantic cod (Gadus morhua) reared at different locations off Norway. Aquaculture 259: 66-73. 